Running Title: Dissociation of lipid transfer activities of MTTP

\title{
A missense mutation dissociates triglyceride and phospholipid transfer activities in zebrafish and human microsomal triglyceride transfer protein
}

\author{
Meredith H. Wilson ${ }^{1}$, Sujith Rajan ${ }^{2}$, Aidan Danoff ${ }^{1,3}$, Richard J. White ${ }^{4,5}$, Monica R. Hensley ${ }^{1}$, \\ Vanessa H. Quinlivan', James H. Thierer ${ }^{1,3}$, Elisabeth M. Busch-Nentwich ${ }^{4,5}$, \\ M. Mahmood Hussain ${ }^{2,}$, Steven A. Farber ${ }^{1,3,{ }^{*}}$
}

1 Carnegie Institution for Science Department of Embryology, Baltimore, MD 21218

132 New York University Winthrop Hospital, Mineola, NY11501

143 Johns Hopkins University Department of Biology, Baltimore, MD 21218

4 Wellcome Sanger Institute, Wellcome Genome Campus, Hinxton, Cambridge, CB101SA, UK

5 Department of Medicine, University of Cambridge, Cambridge, CB2 OQQ, UK

Footnote:

${ }^{*}$ Corresponding Author:

Steven A. Farber

M. Mahmood Hussain farber@carnegiescience.edu

Mahmood.Hussain@nyulangone.org

\section{SUMMARY}

Microsomal triglyceride transfer protein (MTP) transfers triglycerides and phospholipids and is essential for the assembly of Apolipoprotein $B(A p o B)$-containing lipoproteins in the endoplasmic reticulum. We have discovered a zebrafish mutant $\left(m t t p^{c 655}\right)$ expressing a Cterminal missense mutation (G863V) in Mttp, one of the two subunits of MTP, that is defective at transferring triglycerides, but retains phospholipid transfer activity. Mutagenesis of the conserved glycine in the human MTTP protein (G865V) also eliminates triglyceride but not phospholipid transfer activity. The G863V mutation reduces the production and size of ApoBcontaining lipoproteins in zebrafish embryos and results in the accumulation of cytoplasmic lipid droplets in the yolk syncytial layer. However, $m t^{t} p^{c 65}$ mutants exhibit only mild intestinal lipid malabsorption and normal growth as adults. In contrast, zebrafish mutants bearing the previously identified $m t t p^{\text {stl }}$ mutation (L475P) are deficient in transferring both triglycerides and phospholipids and exhibit gross intestinal lipid accumulation and defective growth. Thus, the G863V point mutation provides the first evidence that the triglyceride and phospholipid transfer

40 functions of a vertebrate MTP protein can be separated, arguing that selective inhibition of the

41 triglyceride transfer activity of MTP may be a feasible therapeutic approach for dyslipidemia. 
Keywords: MTP, MTTP, ApoB, lipid transfer, zebrafish, yolk

43

\section{INTRODUCTION}

In vertebrates, Apolipoprotein B-containing lipoproteins (B-Ips) are produced by the intestine and liver and transport lipid and fat-soluble vitamins to the peripheral tissues through the circulation. Lipoproteins are composed of a neutral core of triglyceride (TG) and cholesteryl esters surrounded by a monolayer of phospholipid, free cholesterol and sphingomyelin. B-Ips contain one apolipoprotein $B(A p o B)$ scaffold protein embedded in the phospholipid monolayer as well as other exchangeable lipoproteins (Hussain et al., 1996; Schumaker et al., 1994). B-Ip assembly occurs in the endoplasmic reticulum (ER) and requires the activity of microsomal triglyceride transfer protein (MTP) (Hussain et al., 2003b; Patel and Grundy, 1996; Wetterau and Zilversmit, 1986). As ApoB is translated and translocated into the lumen of the ER, MTP physically interacts with and transfers lipids to $A p o B$ to form primordial lipoproteins (Bradbury et al., 1999; Hussain et al., 2003b; Patel and Grundy, 1996; Wu et al., 1996). MTP may also aid in the formation of TG-rich lumenal lipid droplets that are believed to fuse to the primordial lipoproteins, thus increasing the size of nascent lipoproteins (Alexander et al., 1976; Boren et al., 1994; Hamilton et al., 1998; Kulinski et al., 2002; Raabe et al., 1999; Wang et al., 1997).

MTP is a heterodimer of the large $97-\mathrm{kDa}$ M subunit (microsomal triglyceride transfer protein, MTTP) and the small 58-kDa P subunit protein disulfide isomerase (PDI)(Wetterau et al., 1990). Mutations in the MTTP gene that prevent lipid transfer and ApoB secretion cause the disease Abetalipoproteinemia (OMIM 200100), characterized by a virtual absence of plasma B-Ips (Kane, 1995; Sharp et al., 1993; Shoulders et al., 1993; Wetterau et al., 1992). Patients exhibit fat malabsorption, low plasma triglyceride, and cholesterol levels as well as fat-soluble vitamin deficiencies (Kane, 1995; Lee and Hegele, 2014; Walsh and Hussain, 2016). Without adequate supplementation of essential fatty acids and fat-soluble vitamins, these patients can develop a variety of complications including neurological, opthalmological, and hematological disorders (Kane, 1995; Lee and Hegele, 2014).

Vertebrate MTP can transfer triacylglycerol, diacylglycerol, phospholipid, cholesteryl ester, ceramide, and sphingomyelin between vesicles in vitro (Athar et al., 2004; Iqbal et al., 2015; Jamil et al., 1995; Rava et al., 2005; Wetterau and Zilversmit, 1984, 1985). Kinetic studies suggest that MTP transiently interacts with membranes, acquires lipids, and then delivers these 
76 lipids to an acceptor membrane. The transfer of lipids occurs down a concentration gradient

77 and does not require energy (Atzel and Wetterau, 1993, 1994). While vertebrate MTP

78 predominantly transfers TG (Rava et al., 2005; Wetterau and Zilversmit, 1985), the Drosophila

79 orthologue of MTP lacks TG transfer activity (Rava et al., 2006), has phospholipid transfer

80 activity and supports secretion of vertebrate ApoB (Khatun et al., 2012; Rava and Hussain,

81 2007; Rava et al., 2006; Sellers et al., 2003). A further analysis of MTTP orthologues in

82 divergent species, including nematodes, insects, fish, and mammals, indicates that all

83 orthologues bind PDI, localize to the ER, and support human ApoB secretion (Rava and

84 Hussain, 2007). However, only vertebrate MTP orthologues exhibit TG transfer activity,

85 suggesting that phospholipid transfer activity was the original function of MTP orthologues and

86 that neutral lipid transfer first evolved in fish (Rava and Hussain, 2007).

Here we describe a hypomorphic missense mutation in the C-terminal domain of zebrafish $m t t p$ (G863V) that decreases the production and size of B-Ips in vivo, but has minimal effects on lipid malabsorption in the intestine and no effect on growth. Biochemical characterization of the G863V allele indicates that it is defective in triglyceride transfer activity, but retains phospholipid transfer activity. Further, we show that mutation of the conserved glycine at position 865 in human MTTP also selectively abolishes triglyceride transfer activity. Taken together, these data provide the first evidence that the lipid transfer functions of a vertebrate MTP can be biochemically dissociated and argues that phospholipid transfer activity is sufficient for absorption of dietary lipid.

\section{RESULTS}

The c655 allele is a missense mutation in mttp. During routine screening, we identified

102 zebrafish embryos with opaque yolks (Figure 1A). In contrast to the translucent yolks of wild-

103 type fish, when illuminated by transmitted light, the mutant yolks appear dark (Figure 1A) and,

104 with incident light, appear off-white (Figure 1- figure supplement 1A). The phenotype was

105 found to be present in Mendelian ratios (Figure 1A), suggesting the presence of a homozygous

106 recessive mutation. This new allele is identified as Carnegie allele c655. To map the location of

107 the causative mutation, we did RNA sequencing of 23 opaque-yolk c655 mutants and 23

108 translucent-yolk siblings and performed a Euclidean distance mapping analysis using the

109 Mutation Mapping Analysis Pipeline for Pooled RNA-seq (MMAPPR)(Hill et al., 2013). The 
110 Loess fit to the mapping scores (Euclidean Distance ${ }^{4}$ ) (Figure 1B, top) indicated the c655

111 mutation was located on Chromosome 1 and lay in the region between 9-20 MB (Figure 1B,

112 bottom). Single nucleotide variants (SNVs) present in this 11MB region in c655 mutant embryos

113 were assessed for their effect on annotated genes using the Ensembl Variant Effect Predictor

114 (McLaren et al., 2016), including using the Sorting Intolerant from Tolerant algorithm (SIFT)

115 (Sim et al., 2012), to predict the impact of changes on protein-coding sequence (tolerated or

116 deleterious). We extracted variants that alter the protein-coding sequence as candidates for the

117 causal mutation (223 variants in 64 genes, of which 42 are missense variants predicted to be

118 deleterious; Supplementary File 1).
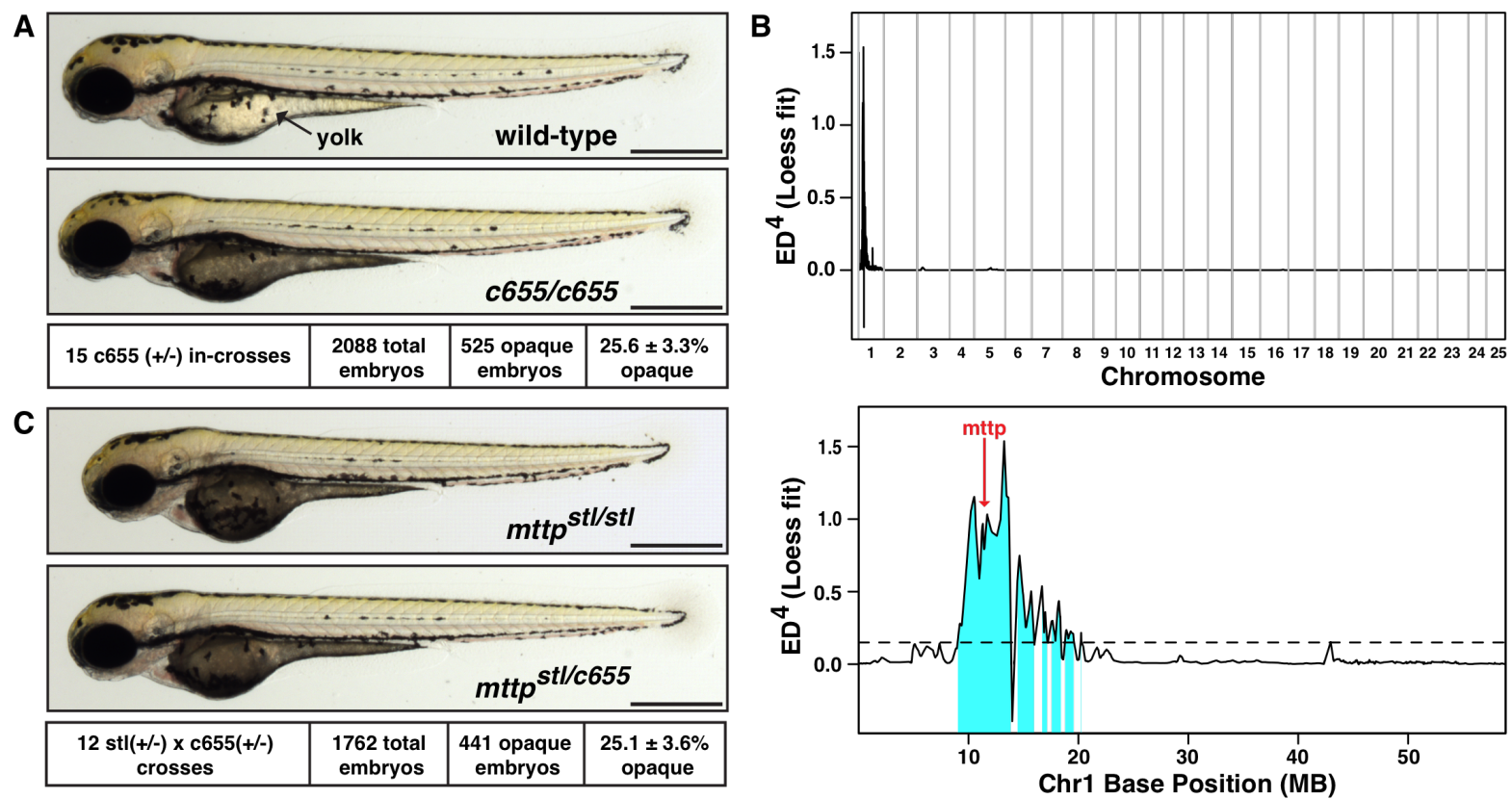

D mttp: Chromosome 1: 11,419,101-11,440,589, reverse strand

120

121

122

123

124

125

126

127

\section{Figure 1: The $c 655$ allele is a missense mutation in the M-subunit of microsomal}

triglyceride transfer protein. (A) Representative images of wild-type (top) and homozygous mutant (middle) c655 zebrafish embryos at 3 days post fertilization (dpf); Scale $=500 \mu \mathrm{m}$. The dark/opaque yolk phenotype segregated with a Mendelian ratio consistent with a homozygous recessive mutation (bottom), mean $+/-\mathrm{SD}$.

(B) Euclidean distance mapping analysis plots 
128 produced by MMAPPR (Hill et al., 2013), showing the likely genomic region of the c655

129 mutation: plot of the LOESS fit to the mapping scores (Euclidean Distance ${ }^{4}$ ) across all 25

130 chromosomes (top) and expanded view of chromosome 1(GRCz10: CM002885.1)(bottom).

131 Red arrow shows the position of the $\mathrm{G}>\mathrm{T}$ missense mutation in mttp (ENSDARG00000008637,

132 position 11,421,261(GRCz10). (C) Representative images of a homozygous mutant zebrafish

133 embryo carrying the previously described stalactite (stl) missense mutation in mttp (Avraham-

134 Davidi et al., 2012)(top) and a trans-heterozygous $m t t p^{\text {st/ } c 655}$ embryo (middle); 3 dpf, scale $=500$

$135 \mu \mathrm{m}$. The dark/opaque yolk phenotype is present at expected ratios (bottom) and genotyping

136 confirms only embryos with opaque yolks are trans-heterozygous for the mttp alleles. (D)

137 Depiction of the mttp gene structure highlighting the locations of the stl (L475P) (position

138 11,431,645, GRCz10, transcript mtp-204 (ENSDART00000165753.2) nucleotide 2588) and

$139 c 655$ (G863V) (nucleotide 1424) missense alleles in exon 11 and 18, respectively.

142

143

144

145

146

147

148
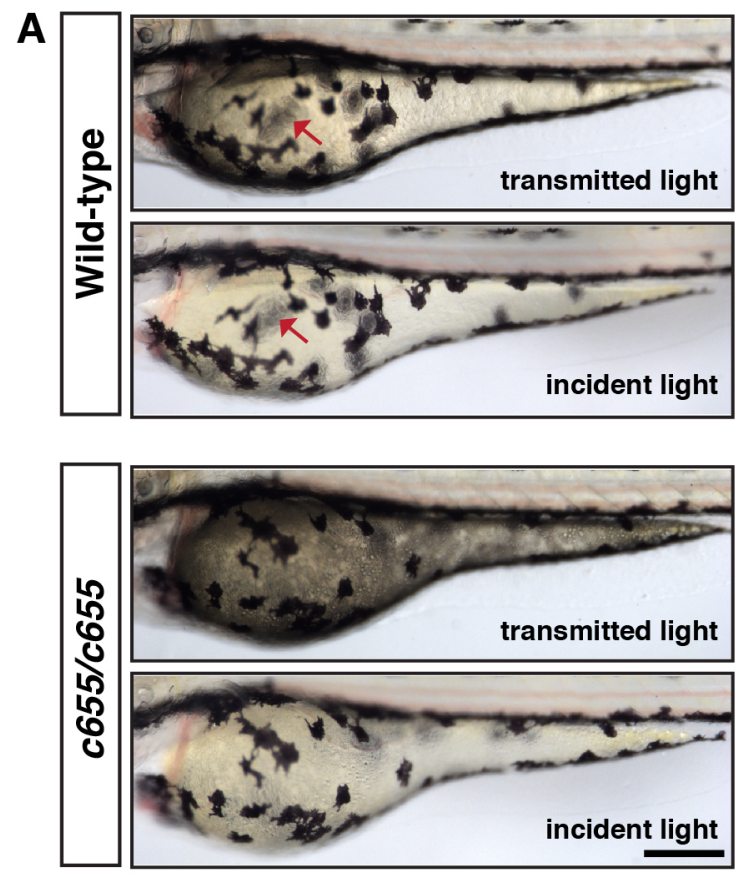

Figure 1 - figure supplement 1: Lipid droplets block light transmission through the yolk of the embryo. (A) Wild-type and c655 mutant embryos were imaged at $3 \mathrm{dpf}$ using either transmitted light (illumination below the fish) or incident light (illumination from above the fish). The wild-type embryos are translucent; the pigment cells on the opposite side of the embryo (red arrow) are visible through the yolk with both light sources. The yolk is opaque in the 
mutants; it appears dark with transmitted light and white with incident light. Pigment cells on the opposite side of the embryo are barely visible in mutant embryos, regardless of light source.

$151 \quad$ Scale $=200 \mu \mathrm{M}$.

154 One of the SNVs linked to the 6555 phenotype was a missense mutation predicted to be 155 deleterious in exon 18 of the microsomal triglyceride transfer protein gene

156 (ENSDARG00000008637, Chr1:11,421,261 GRCz10). A previously identified missense

157 mutation in exon 11 of zebrafish $m t t p$, stalactite (stl), also presents with an opaque yolk

158 phenotype (Figure 1C, top) (Avraham-Davidi et al., 2012), suggesting that the c655 opaque yolk

159 phenotype might result from this newly identified missense mutation in mttp. To test this

160 hypothesis, we performed complementation crosses between $\mathrm{mttp}^{\mathrm{c655/+}}$ heterozygous fish and

$161 \mathrm{mttp}{ }^{s t /+}$ heterozygous fish. The $c 655$ mutation failed to complement the $m t t p^{s t l}$ mutation, as one-

162 quarter of the embryos in these crosses displayed the opaque yolk phenotype (Figure 1C,

163 bottom). All of the embryos exhibiting opaque yolks were heterozygous for both the $\mathrm{mttp}^{\text {stl }}$ and

164 the c655 mutation in mttp. This strongly argues that the G/T SNV in exon 18 of $m t t p$ is the

165 causative allele for the c655 opaque yolk phenotype. This was further confirmed by rescuing

166 the c655 opaque yolk phenotype with injections of a wild-type mttp-FLAG plasmid at the 1-cell

167 stage (Figure 1- figure supplement 2).

168

A c655/c655 injected with EGFP-CAAX

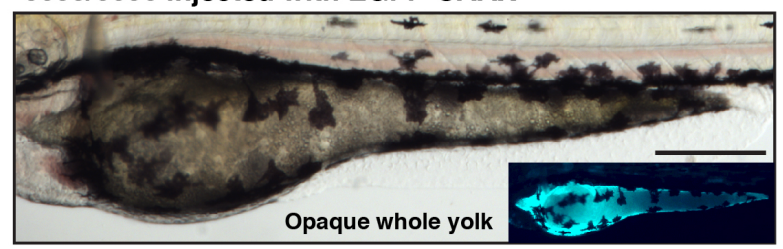

C

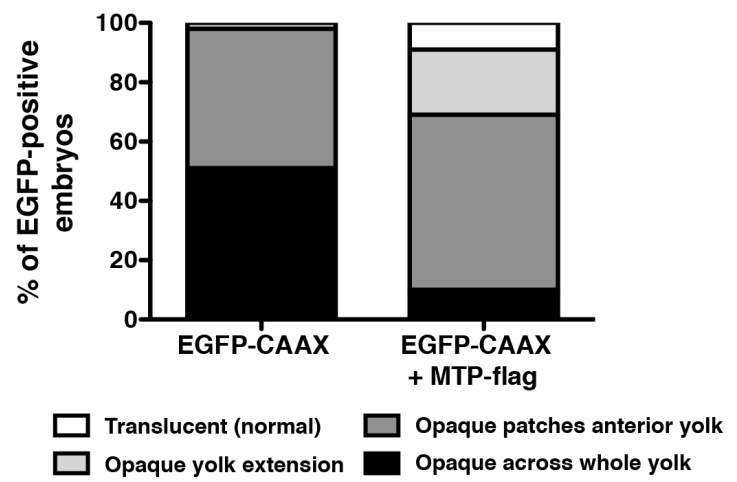

B c655/c655 injected with EGFP-CAAX + mttp-flag
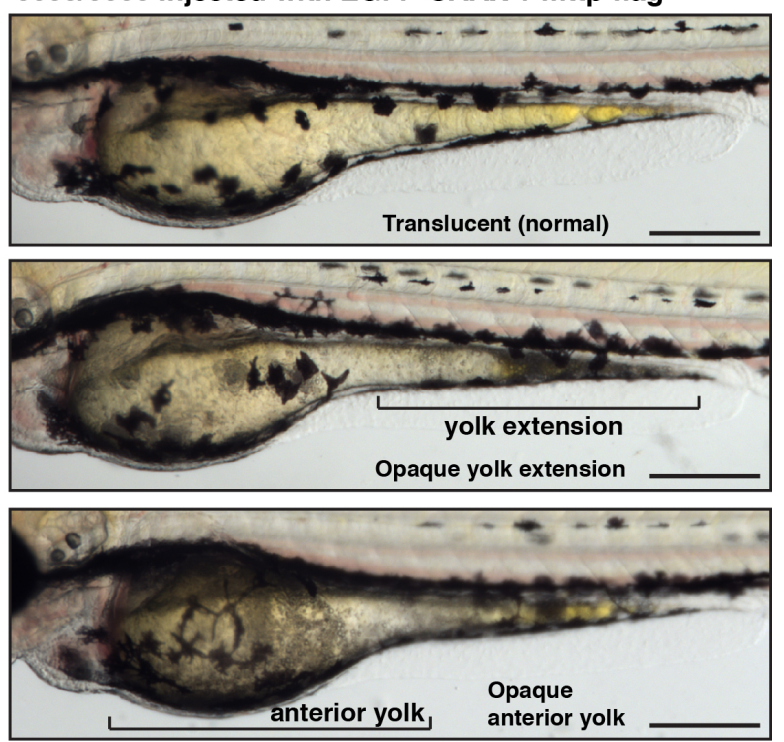
172 Figure 1 - figure supplement 2: Expression of wild-type zebrafish mttp-FLAG rescues

173 the opaque yolk phenotype in $\boldsymbol{m t t p}{ }^{c 655 / c 655}$ embryos. One-cell stage $m t t p^{c 655 / c 655}$ embryos

174 were co-injected with CMV: mttp-FLAG and the CMV: eGFP-CAAX plasmid, or CMV: eGFP-

$175 C A A X$ alone as a control. Embryos expressing eGFP in the YSL were imaged at $3 \mathrm{dpf}$, and

176 images were blinded and scored for the degree of yolk opacity. (A) Representative image of an

$177 m t p^{c 655 / c 655}$ mutant embryo expressing eGFP-CAAX in the YSL and a fully opaque yolk. (B)

178 Examples of injected embryos with varying degrees of yolk opacity (normal translucent yolk,

179 opaque region in the yolk extension, opaque patches in the anterior yolk with or without opaque

180 yolk extension). (C) Images were binned into the four noted categories of yolk opacity. Results

181 represent pooled data from 3 independent experiments, $n=91$ control and 102 Mttp-FLAG

182 eGFP-positive embryos total. Chi-square test, $\mathrm{p}<0.001$. Scale $=500 \mu \mathrm{M}$.

185 Both the $m t t p^{s t l}$ allele and $m t t p^{c 655}$ allele are missense mutations. The stl allele results in the 186 conversion of a leucine to a proline at residue 475 and the c655 mutation is a glycine to valine 187 mutation in the $\mathrm{C}$-terminus of the protein at residue 863 (total length $=884$ residues) (Figure

188 1D). An additional SNV in mttp at position Chr1:11,421,300 GRCz10 (T/C) causing a missense 189 mutation (M850T) was identified in c655 mutants; however, this SNP was not predicted to be 190 deleterious and has been previously noted in the Ensembl zebrafish genome database.

191 Furthermore, no change in mRNA expression was noted for $m t t p$ in the mttp $^{c 655}$ mutants in our 192 RNAseq data-set (log2[fold change] $=0.18$, adj. $p$-value $=0.19$ ).

194 Although the $m t t p^{\text {st/stl }}, m t t p^{c 655 / c 655}$, and trans-heterozygous $m t t p^{s t / c 655}$ fish all exhibit opaque 195 yolks, the $m t t p^{\text {st/stl }}$ mutants have a more severe phenotype, in that their yolks are darker and

196 they retain the opaque phenotype longer during development. The mttp ${ }^{c 655 / c 655}$ mutant

197 phenotype is the least severe and the trans-heterozygotes exhibit an intermediate phenotype

198 (Figure 1- figure supplement 3). Encouraged by the differences in embryonic phenotype, we

199 hypothesized that these mutations would provide an opportunity to further dissect the molecular 200 details of MTP function in vivo. 

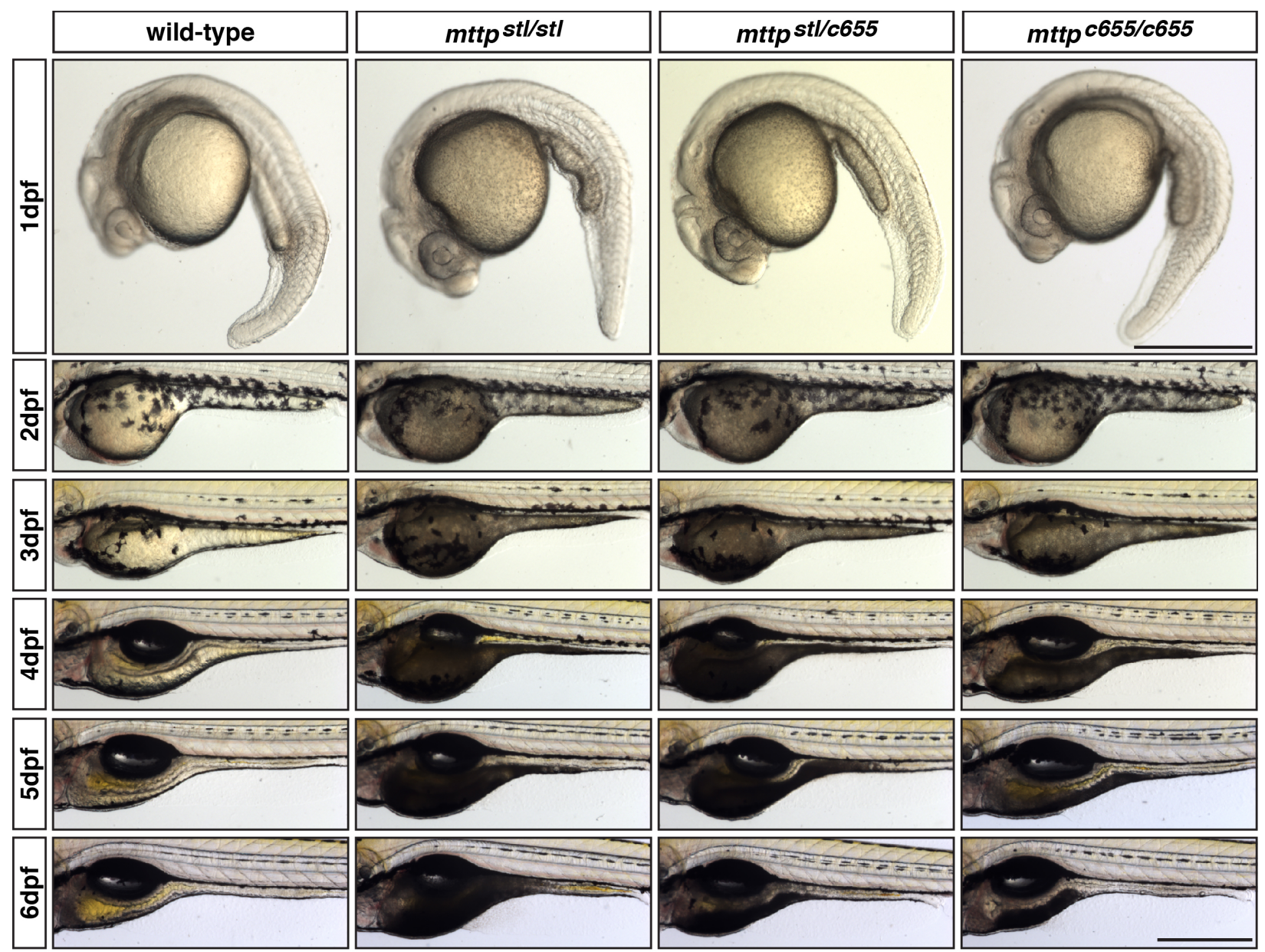

Figure 1 - figure supplement 3: The stl and c655 mttp mutations have differential effects on the degree of yolk opacity during embryonic development. Representative

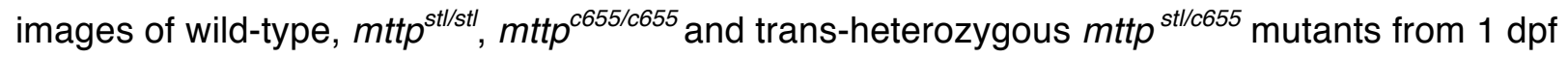
to $6 \mathrm{dpf}$. The $m t t \mathrm{p}^{\text {st/stl }}$ mutants are visibly opaque at $1 \mathrm{dpf}$ and the area of opacity is retained for longer than in $m t t p^{\text {st/c655 }}$ or $m t t p^{c 655 / 6655}$ mutants. 3 dpf images are the same fish shown in Figure

210 1. Scale $=500 \mu \mathrm{M}$.

213 Mttp mutants accumulate cytoplasmic lipid droplets in the yolk syncytial layer. As a

214 lecithotrophic organism, zebrafish rely on their maternally-derived yolk as the source of nutrients

215 and building blocks for embryogenesis (Hiramatsu et al., 2015; Mani-Ponset et al., 1996;

216 Vernier and Sire, 1977). The yolk is rich in lipids (Fraher et al., 2016; Miyares et al., 2014;

217 Wiegand, 1996) and following lipolysis and re-esterification, the lipids are packaged into

218 lipoproteins in the ER of the yolk syncytial layer (YSL), a multi-nucleated cytoplasm that 
219 surrounds the yolk mass (Figure 2A)(Carvalho and Heisenberg, 2010; Kimmel and Law, 1985;

220 Walzer and Schonenberger, 1979a, b). The zebrafish YSL expresses apolipoprotein B (Otis et 221 al., 2015) and microsomal triglyceride transfer protein (Marza et al., 2005; Schlegel and Stainier, 222 2006). The YSL produces B-Ips (Thierer et al., In Press; Vernier and Sire, 1977; Walzer and

223 Schonenberger, 1979a), similar to the intestine (Glickman et al., 1976; Kessler et al., 1970),

224 liver (Mahley et al., 1970), placenta (Madsen et al., 2004), and embryonic yolk sac in mammals

225 (Farese et al., 1996; Plonne et al., 1992). In the mammalian intestine, ApoB mRNA is edited, 226 resulting in a truncated ApoB molecule (ApoB48)(Davidson and Shelness, 2000; Kane et al., 227 1980); however, there is no evidence for editing in zebrafish, so all B-lp-producing tissues,

228 including the YSL, secrete lipoproteins containing ApoB100 (Thierer et al., In Press).

230 When MTP is mutated or absent, B-Ip production is reduced or absent and TG accumulates in 231 cytoplasmic lipid droplets (LDs) instead (Khatun et al., 2012; Raabe et al., 1999). We have 232 previously shown that accumulation of LDs in intestinal enterocytes of zebrafish larvae fed a 233 high-fat meal causes the gut to be opaque (Otis and Farber, 2016)(Figure 2 - figure supplement 234 1), most likely due to the lipid droplets' ability to scatter light (Hwang et al., 2018; Michels et al., 235 2008). Therefore, we hypothesized that the yolk opacity in the mttp mutant embryos is due to 236 aberrant accumulation of LDs in the cytoplasm of the YSL. Using transmission electron

237 microscopy, we found that the YSL in the wild-type embryos contains very few, if any, canonical 238 YSL LDs, whereas the $m t t p^{\text {st/stl }}, m_{t t} p^{c 655 / c 655}$ and trans-heterozygous $m t t p^{s t / c 655}$ embryos

239 accumulate substantial numbers of cytoplasmic LDs (Figure 2B). LDs in $m t t p^{\text {st/stl }}$ mutants are 240 more numerous and more uniform in size, whereas the $m t t p^{6655 / 6655}$ mutants often had very large 241 LDs in addition to small droplets (Figure 2C). As a result, the number of LDs per area of the

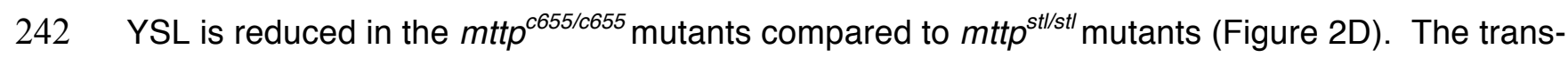
243 heterozygous fish had LDs that were more similar in size to the $\mathrm{mttp}^{\text {st/stl }}$ mutants and had a trend 244 toward fewer lipid droplets per YSL area, although this was not significant. 

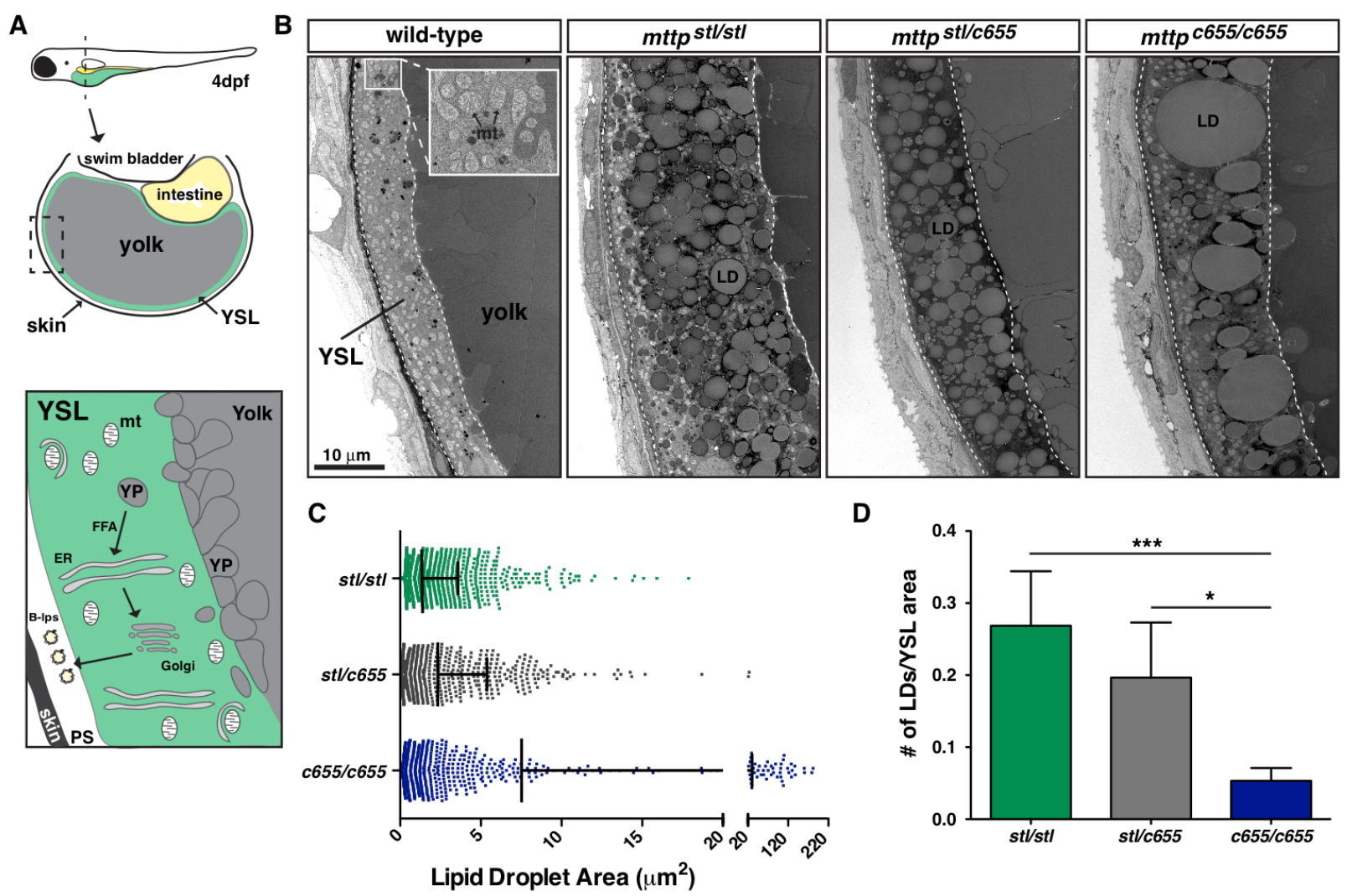

Figure 2: The opaque yolk phenotype results from the accumulation of aberrant cytoplasmic lipid droplets in the yolk syncytial layer. (A) (Top) Cartoon depicting the crosssectional view of a 4-dpf zebrafish embryo. The yolk syncytial layer (YSL) surrounds the yolk mass and serves as the embryonic digestive organ. The dashed box indicates the view expanded in the bottom panel and in panel B. (Bottom) Stored yolk lipids undergo lipolysis in yolk platelets (YP), presumably releasing free fatty acids into the YSL. These fatty acids are re-

254 esterified in the ER bilayer to form triglycerides, phospholipids, and cholesterol esters. The 255 lipids are packaged into ApoB-containing lipoproteins in the ER with the help of MTP and are 256 further processed in the Golgi before being secreted into the perivitelline space (PS) and then 257 circulation. (B) Representative transmission electron micrographs of the yolk and YSL from wild258 type and $m t t p$ mutants; dashed lines delineate the $\mathrm{YSL}$ region, $\mathrm{mt}=$ mitochondria, $\mathrm{scale}=10$ $259 \mu \mathrm{m}$. (C) Quantification of lipid droplet size in mttp mutants, $\mathrm{n} \geq 700$ lipid droplets in 2 fish per 260 genotype; mean +/- SD. (D) Quantification of the number of lipid droplets per YSL area, $n=7-9$ 261 YSL regions per genotype (3-5 regions per fish, 2 fish per genotype); mean +/- SD, Kruskall262 Wallis with Dunn's Multiple Comparison test, vs. mttp ${ }^{c 655 / c 655 *} p<0.05,{ }^{* \star \star} p<0.001$. 
A

B

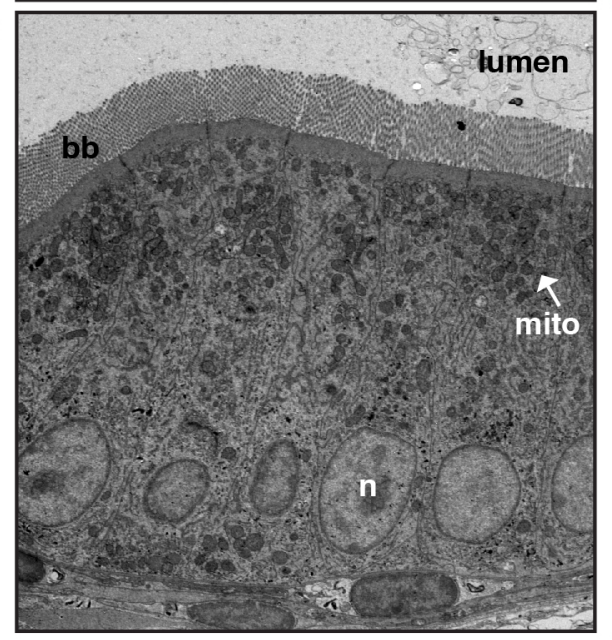

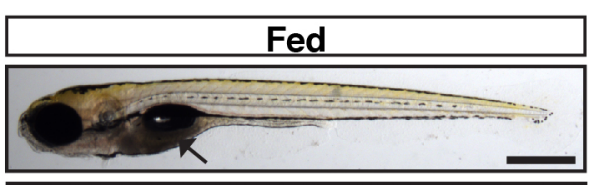

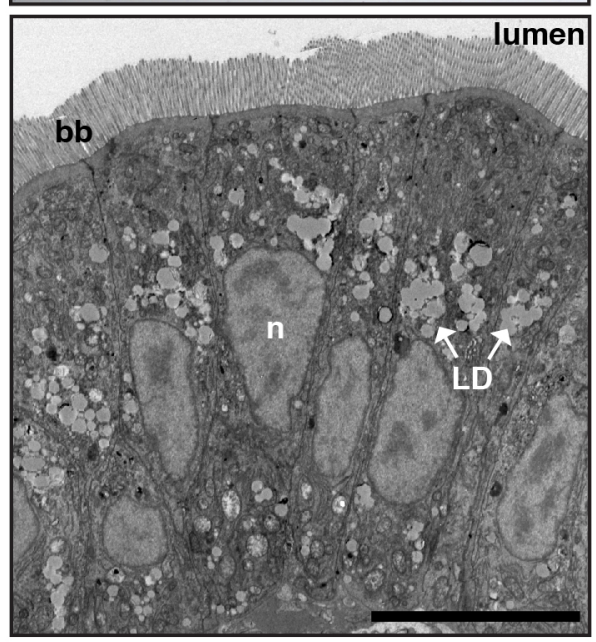

Figure 2 - figure supplement 1: Lipid droplets block light transmission through the larval intestine. (A) Wild-type fish at $6 \mathrm{dpf}$ were fed a high-fat meal for $1 \mathrm{~h}$ as described previously (Otis and Farber, 2016). Unfed fish have translucent intestines (black arrow, left) when imaged with transmitted light, whereas fed fish have opaque intestines (black arrow, right). Scale $=500$ $\mu \mathrm{M}$. (B) Electron microscopy following a $1 \mathrm{~h}$ high-fat feed reveals an accumulation of cytoplasmic lipid droplets in the intestinal enterocytes. By scattering light and blocking light transmission through the intestine, the accumulation of cytoplasmic lipid droplets causes the intestine to appear opaque. Nucleus (n), mitochondria (mito), brush border (bb), lipid droplet (LD). Scale $=10 \mu \mathrm{M}$.

\section{The stl and c655 mutations have differential effects on adult size and steatosis. Patients}

with Abetalipoproteinemia often present in infancy with growth retardation, diarrhea, fat malabsorption, and failure to thrive (reviewed in (Lee and Hegele, 2014)), and whole body deficiency of MTP in a murine model is embryonic lethal (Raabe et al., 1998). In agreement with the mouse phenotype, in the original characterization of the zebrafish $\mathrm{mttp}^{\text {st/stl }}$ phenotype it was noted that the fish did not survive past 6 days post fertilization (dpf)(Avraham-Davidi et al., 2012). Therefore, we were surprised to find that some of the st/ mutants were able to survive past larval stages. While these fish are generally much smaller in length and mass (Figure $3 A, B)$ and their viability is reduced relative to their siblings (expected $25 \%$, observed $3.8 \%$ 
[5/131 fish] at 7.5 months), some of these fish have survived to be at least 18 months old.

286 Survival rates are better when the mutants are reared separately and are not competing with

287 siblings for food. Although these fish can reproduce, this is rare. In contrast, the $m t t p^{c 65 / c 655}$

288 mutants do not exhibit reduced viability and we did not find any reduction in size or fertility of the

289 c655 mutants compared with siblings (Figure 3A,B). No difference in length or mass was noted

290 in fish trans-heterozygous for $m t t p^{\text {st//c655 }}$ (Figure 3A,B).

291

A

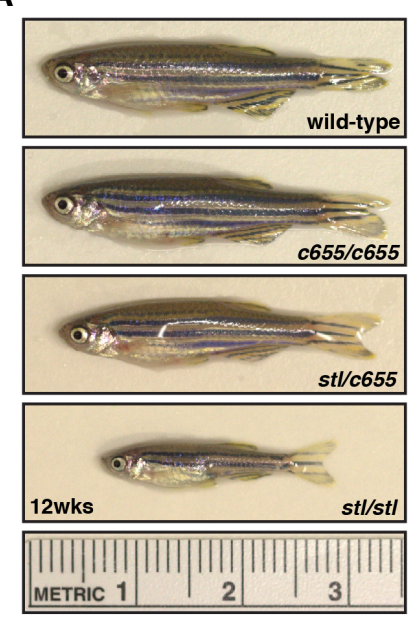

C
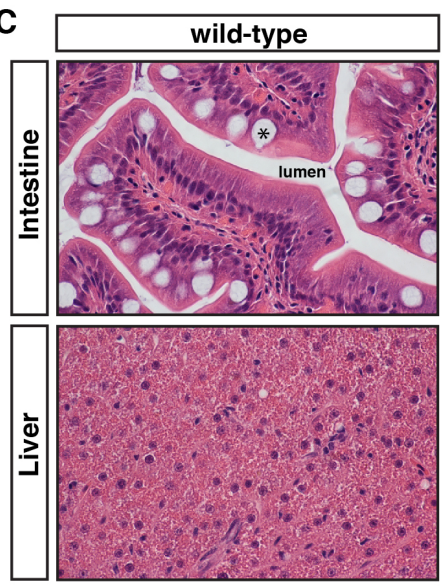

D

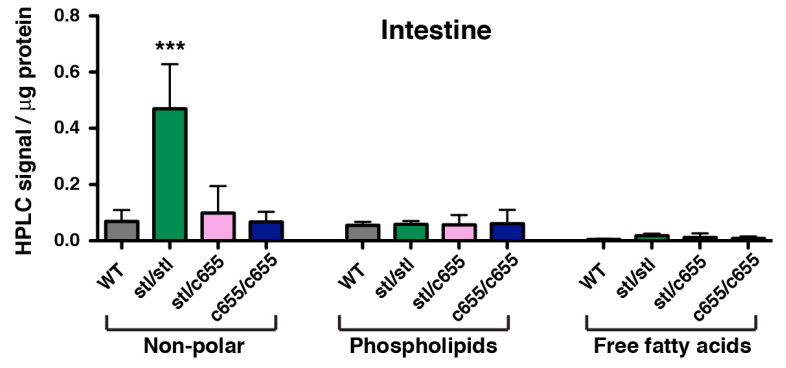

B
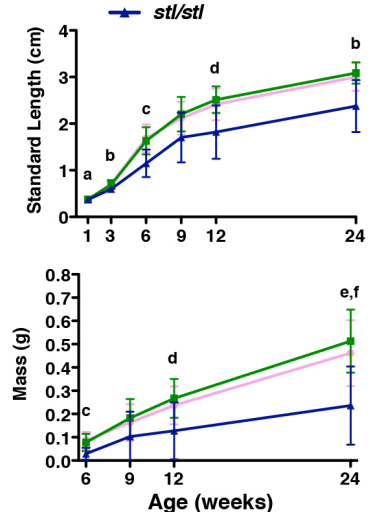
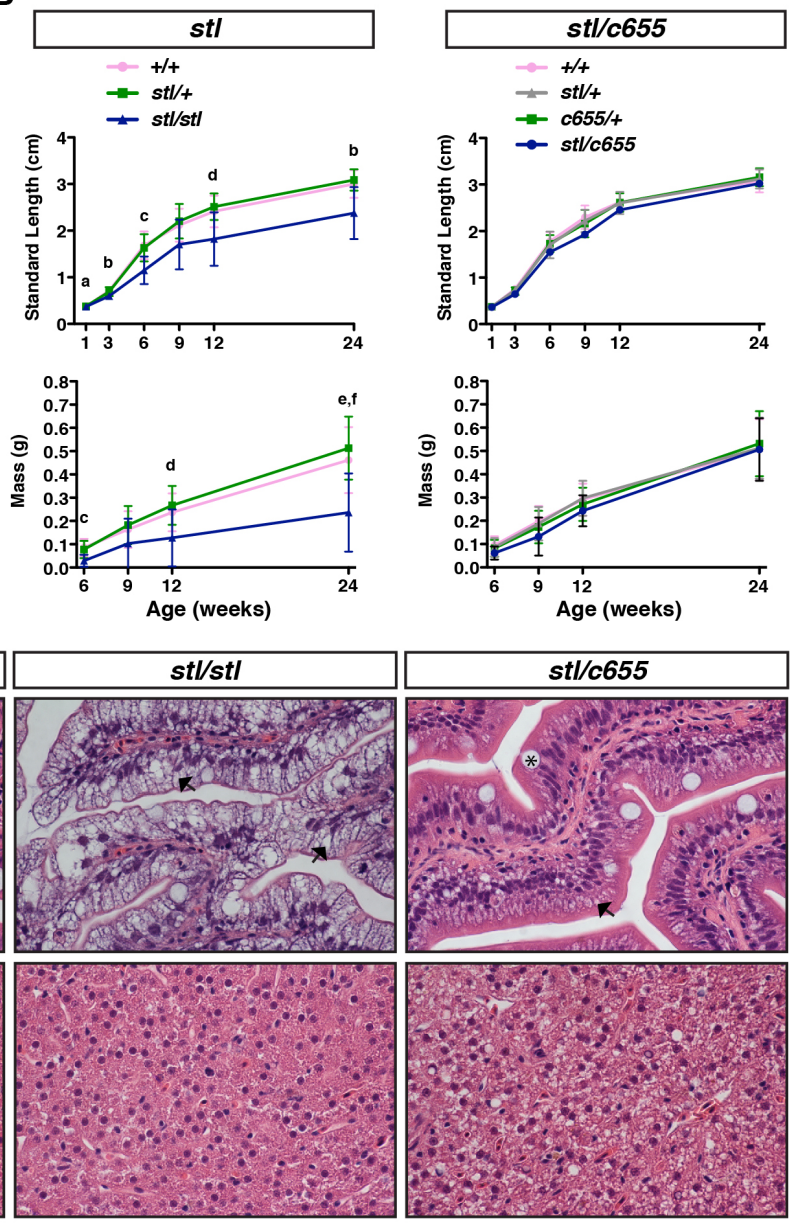

E
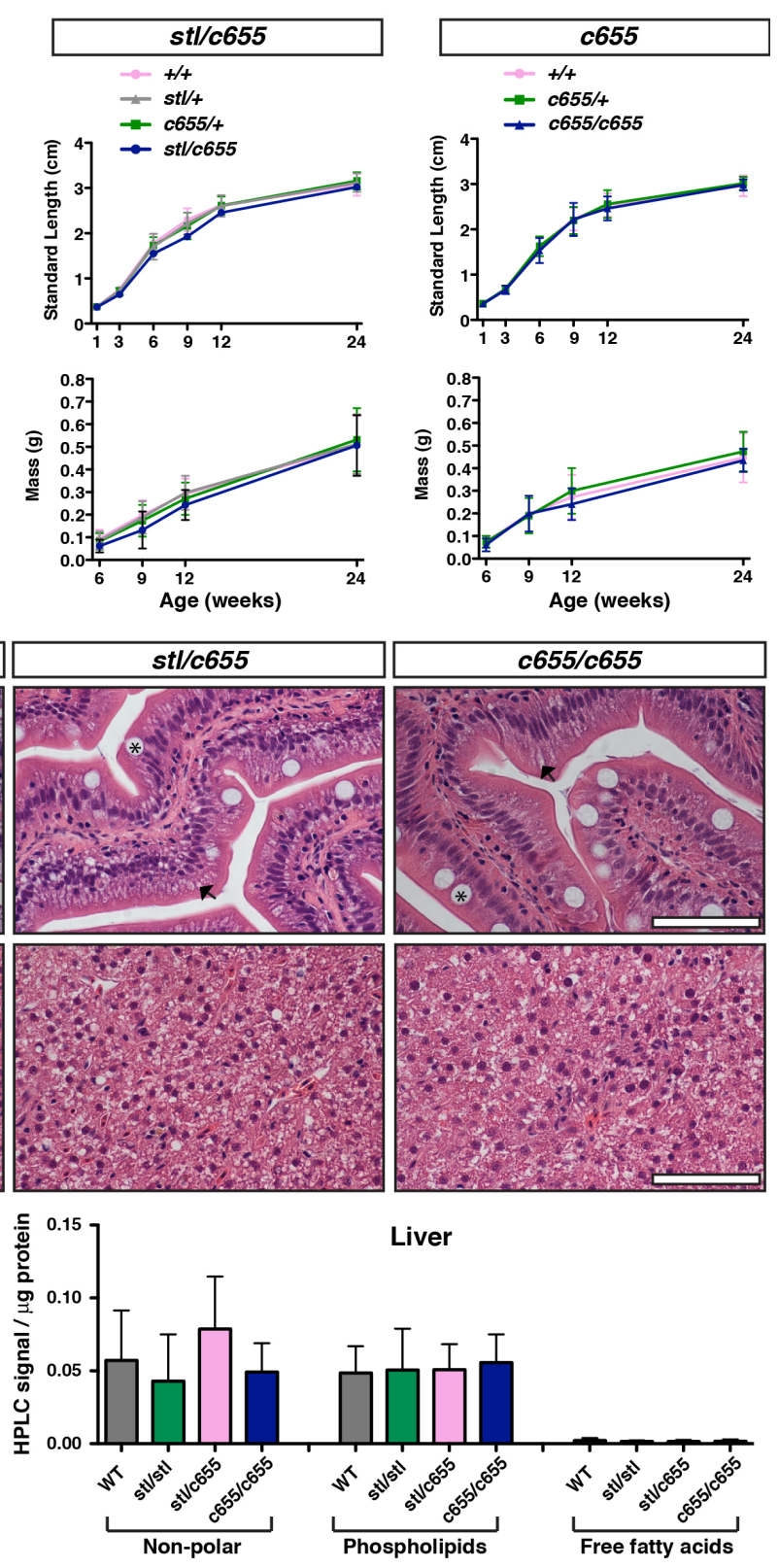

Liver 
294 Figure 3: The stl and c655 mttp mutations have differential effects on the growth and

295 accumulation of lipid in intestine. (A) Representative images of WT and mttp mutant fish at

29612 weeks of age. (B) Developmental time-course of standard length and mass measurements

297 of $m t t p$ mutant fish and siblings. Results are representative of pooled data from two

298 independent experiments, $n=7-80$ fish/genotype/time-point, mean $+/-$ SD. Significance was

299 determined with a Robust ANOVA and Games-Howell post-hoc tests were used to make pair-

300 wise comparisons at each time point. Using a Bonferroni correction, p-values were adjusted to

301 control for multiple comparisons (6 length or 4 mass comparisons), a: $s t l /+v s . s t / / s t l, p<0.01 ; b$ :

$302+$ ++ vs. stl/stl and stl/+ vs. st//stl, p <0.05; c: +/+ vs. stl/stl and stl/+vs. stl/stl, p <0.001; d: stl/+

303 vs. stl/stl, $\mathrm{p}<0.05$; e: $+/+$ vs. st//stl, $\mathrm{p}<0.01$; $\mathrm{f}$ stl/+ vs. stl/stl, $\mathrm{p}<0.001$. (C) Representative

304 images of H\&E stained intestine and liver from adult WT and mttp mutant fish (7.5 months),

305 scale $=50 \mu \mathrm{m},{ }^{*}$ indicate goblet cell, arrows indicate representative lipid accumulation in

306 enterocytes. (D \& E) Quantification of non-polar lipids, phospholipids, and free fatty acids in

307 intestine and liver tissue using high-performance liquid chromatography; $n=5-6$ fish per

308 genotype, mean +/- SD, One-way ANOVA with Bonferroni post-hoc tests were performed

309 separately for each lipid class; ${ }^{* *} p<0.001$ stl/stl vs. all other genotypes.

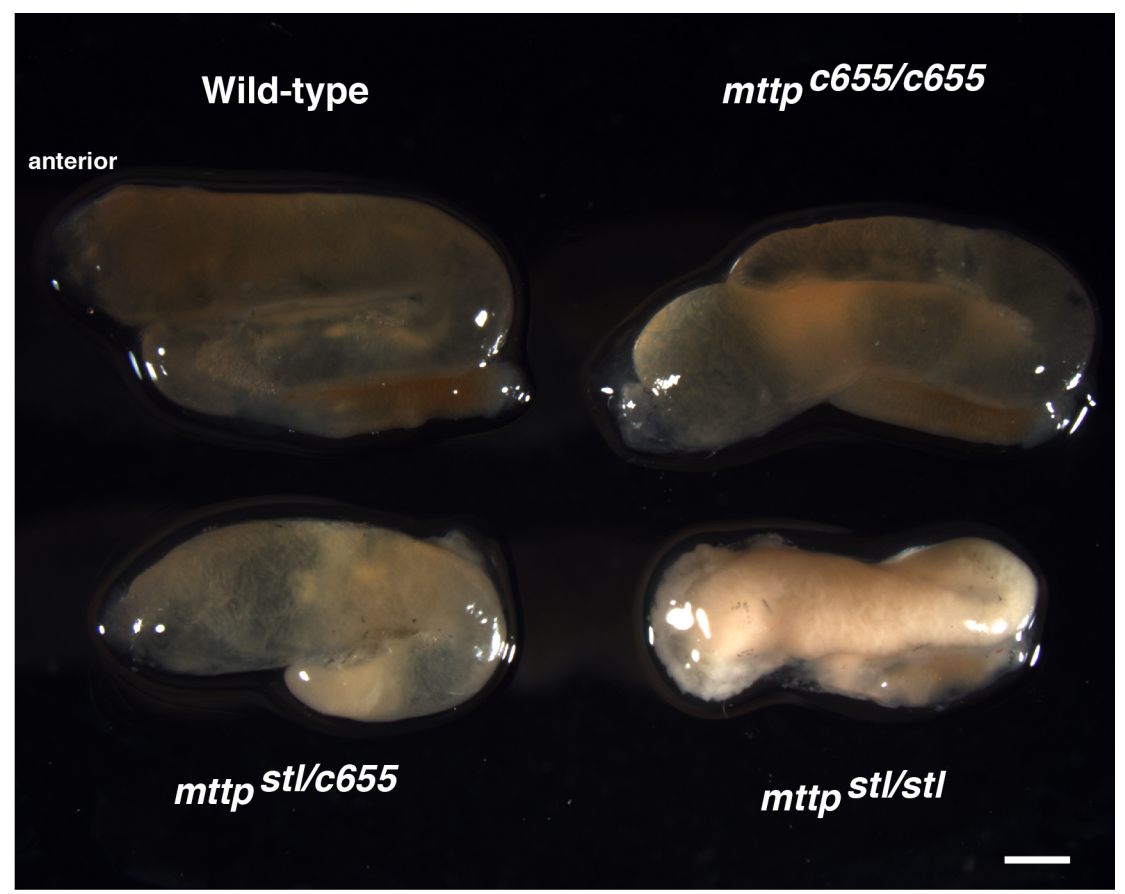


314 Figure 3 - figure supplement 1: Significant lipid accumulation in the intestine of st//stI

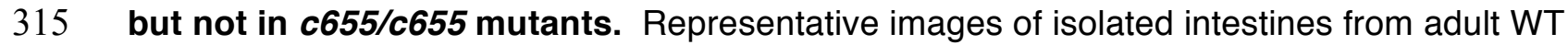

316 and mttp mutant fish (7.5 months), scale $=1 \mathrm{~mm}$.

319 To assess why the $m t t p^{\text {st/stl }}$ mutants, but not the $m t t p^{c 655 / c 655}$ mutants, have defects in growth, we 320 examined whether the mutations have differential effects on fat malabsorption. H\&E staining of 321 intestinal tissue from fasted adults revealed gross accumulation of lipid in the cytoplasm of 322 enterocytes in the $m t t p^{\text {st/stl }}$ fish (Figure 3C, Figure 3 - figure supplement 1 ). The $m t t p^{c 655 / c 655}$

323 mutants were largely protected from this abnormal lipid retention. While the trans-heterozygous

324 fish exhibited more visible lipid retention than either wild-type or $m t t p^{c 655 / c 655}$ fish, this was not as

325 profound as in the $m t t p^{\text {stl/stl }}$ fish. Retention of non-polar lipid (TG and cholesterol ester),

326 phospholipid, and free fatty acids in the intestine were also quantified using HPLC and the

$327 \mathrm{mttp}^{\text {st/stl }}$ mutants contain $\sim 7$ times more neutral lipid than c655 mutants (Figure 3D). This data

328 suggests the growth defects in $m t t p^{\text {stl/stl }}$ mutants result from defects in dietary lipid absorption in

329 the intestine.

331 Besides accumulating lipids in the intestine, Abetaliporoteinemia patients sometimes develop 332 hepatic steatosis (reviewed in (Lee and Hegele, 2014)). Similarly, hepatocyte-specific deficiency 333 of MTTP in mice causes TG and cholesterol to accumulate in the liver (Khatun et al., 2012;

334 Raabe et al., 1999). Therefore, we hypothesized that the adult zebrafish mttp mutants would 335 also exhibit liver steatosis. However, mutants were not different than wild-type (Figure 3C,D).

336 Moreover, there was no significant difference in measured lipids in the livers of the different fish

337 (Figure 3E). While we were surprised that the $m t t p^{\text {st/stl }}$ mutants had very little accumulation of 338 lipid in their livers, this is in agreement with findings that combined intestinal and liver deficiency 339 of Mttp in mice results in accumulation of TG in the intestine, but not in the liver (lqbal et al., 340 2015).

342 Mttp mutations reduce the size and number of ApoB-containing lipoproteins in vivo. To

343 understand how the mttp mutations affect the production and size of B-lps during embryonic

344 development, we crossed the $m t t p^{s t l}$ and $m t t p^{c 655}$ mutations into our LipoGlo reporter line

345 (Thierer et al., In Press). These fish express an in-frame fusion of the engineered luciferase

346 reporter Nanoluc at the C-terminus of the Apolipoprotein Bb.1 gene (Figure 4A). Since ApoB is 
an obligate structural component of B-Ips with only one copy per lipoprotein particle (Elovson et al., 1988), the relative number of tagged lipoprotein particles can be quantified using the NanoGlo assay in extracts from transgenic fish (Thierer et al., In Press). B-lp levels were measured

350 in whole fish lysate throughout embryonic development from 2-6 dpf. During this time, the fish

351 are relying solely on yolk lipids and the $A p \circ B$ quantity measurements reflect lipoprotein particles

352 in the secretory pathway in the YSL, in the circulation, and in cells prior to degradation of

353 endocytosed lipoproteins. Wild-type embryos exhibit an increase in B-Ip particle number from

$354 \quad 2-3 \mathrm{dpf}$ as yolk lipid is packaged into lipoproteins, and then numbers decline as the yolk is

355 depleted and the lipids in the lipoproteins are taken up by target tissues and lipoprotein particles

356 are degraded (Figure 4B). The $m t t p^{6655 / 6655}$ embryos have the same relative number of $A p o B$

357 particles as wild-type embryos at $2 \mathrm{dpf}$, but from 3-6 dpf the numbers of particles never reach

358 wild-type levels and decline more rapidly. In contrast, $m t t p^{\text {st/stl }}$ embryos have profound defects

359 in B-Ip production, since amount of $A p o B$ is significantly lower than wild-type siblings at $2 \mathrm{dpf}$

360 (Figure 4B) (Thierer et al., In Press).

A

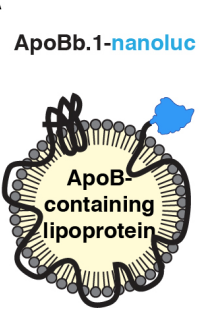

ApoBb.1-nanoluc

B

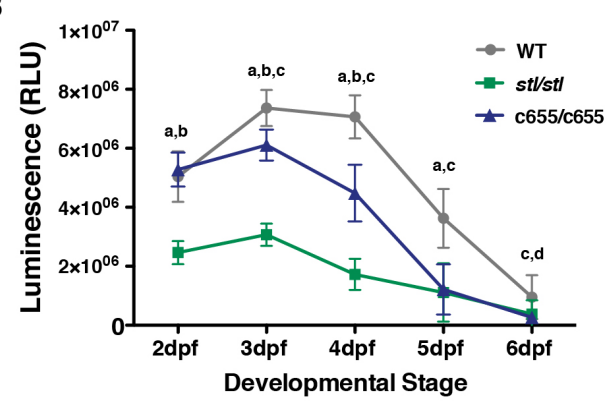

D
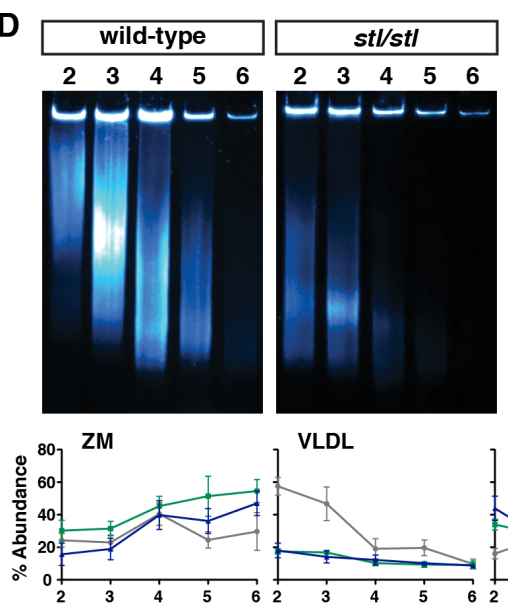

VLDL
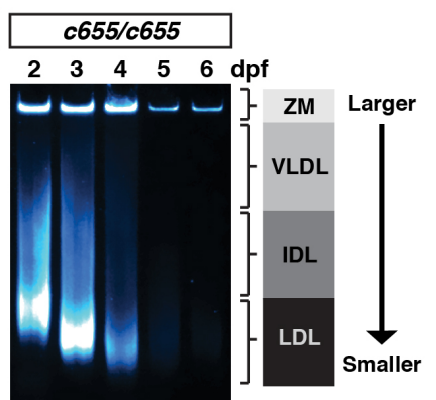

IDL

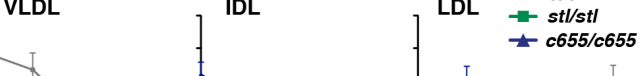

363

$\pm c 655 / c 655$

C
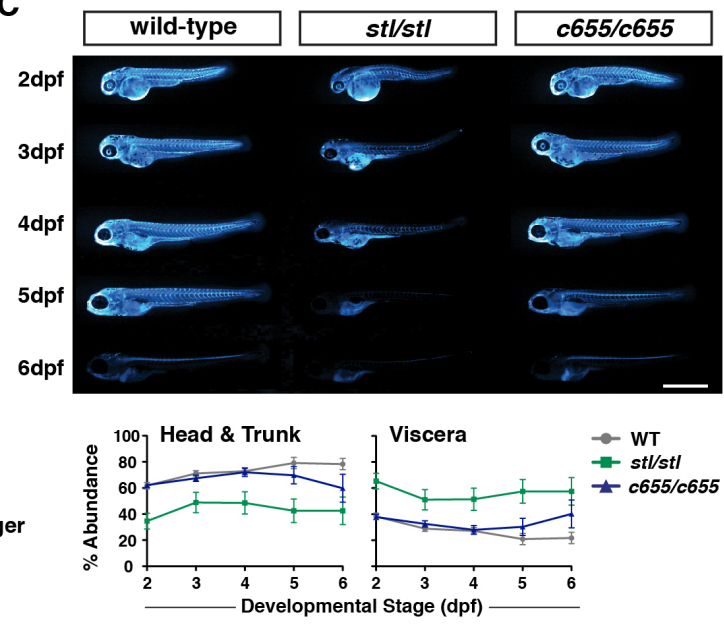

E

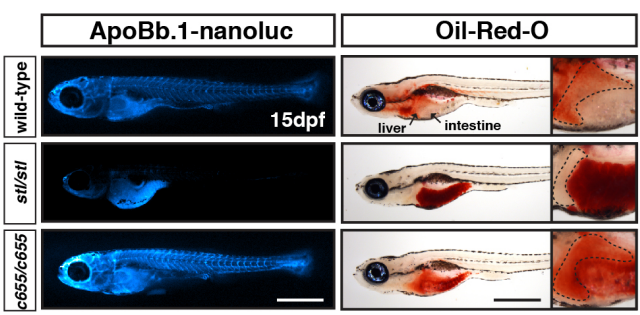


Figure 4: The stl and c655 mttp mutations have differential effects on ApoB-containing lipoprotein number, size and distribution in vivo. (A) LipoGlo fish express the Nanoluc ${ }^{\circledR}$ luciferase enzyme as a C-terminal fusion on ApoBb. 1 as a result of TALEN-based genomic engineering. (B) LipoGlo signal (RLU: relative luminescence units) in WT, ttt $^{\text {st/stl }}$ and $m t t p^{c 655 / c 655}$ fish throughout embryonic development (2-6 dpf). Results represent pooled data from 3 independent experiments, $n=22-34$ fish/genotype/time-point. Significance was determined with a Robust ANOVA, Games-Howell post-hoc tests were performed to compare genotypes at each day of development, and p-values were adjusted to control for multiple comparisons, $\mathrm{a}=\mathrm{WT}$ vs. $m t t p^{\text {st/stl }}, \mathrm{p}<0.001, \mathrm{~b}=\mathrm{mttp}^{\mathrm{c655/6655}}$ vs. $m t t p^{\text {st/stl }}, \mathrm{p}<0.001, \mathrm{c}=\mathrm{WT}$ vs. $m t t p^{c 655 / c 655}, p<0.001, d=W T$ vs. $m t t p^{\text {stl/stl }}, p<0.05$. (C) Representative whole-mount images of B-Ip localization using LipoGlo chemiluminescent microscopy in WT, $m t t p^{\text {st/stl }}$, and $m t t p^{c 655 / c 655}$ fish throughout development; scale $=1 \mathrm{~mm}$. Graphs represent pooled data from 3 independent experiments, $n=13-19$ fish/genotype/time-point; $m t t p^{\text {st/stl }}$ had a significantly different ApoB localization from WT and c655/c655, p<0.001, Robust ANOVA. Post-hoc analysis reveals statistical differences at all developmental stages p<0.05-0.001 (Games-Howell). (D) Representative LipoGlo PAGE gels and quantification of B-Ip size distribution from whole embryo lysates during development. B-Ips are divided into four classes based on mobility,

382 including zero mobility (ZM), and three classes of serum B-Ips (VLDL, IDL and LDL). Graphs

383 show subclass abundance for WT, $m t t p^{\text {st/stl }}$, and $m t t p^{c 655 / c 655}$ fish at each day of embryonic

384 development as described in (Thierer et al., In Press). Results represent pooled data from $n=9$

385 samples/genotype/time-point; at each particle class size, there were statistically significant differences between genotypes (Robust ANOVA, p<0.001). Games-Howell post-hoc analysis revealed numerous differences between genotypes at each developmental stage, see figure supplement 2. (E) Representative whole-mount images of LipoGlo microscopy and Oil Red O imaging in 15-dpf embryos chow-fed for 10 days and fasted $\sim 18$ hrs prior to fixation; scale = 1 $\mathrm{mm}$. Livers (outlined) are magnified for clarity in insets on right. Results represent pooled data from 3 independent experiments, $n=15$ fish/genotype/time-point. To assess the localization of the B-Ips throughout the embryos during development, we fixed

395 the embryos expressing ApoBb.1-nanoluc and performed chemiluminescent whole-mount 396 imaging (Figure 4C). Wild-type and $m t t p^{c 655 / c 655}$ embryos exhibit a similar distribution pattern of 397 LipoGlo throughout 2-4 dpf, but consistent with the quantitative assay, the signal in the head 
398 and trunk decline more rapidly in $m t^{6 t} p^{c 655 / c 655}$ fish. By $6 \mathrm{dpf}$, both wild-type and $m t t p^{c 655 / c 655}$ fish

399 show an accumulation of $A p o B$ in the liver and the spinal cord (Figure 4C) (Thierer et al., In

400 Press). The LipoGlo in the $m t t p^{\text {stlstl }}$ embryos shows a very different pattern, in that it is

401 predominantly localized to the YSL/viscera at all stages and is at very low levels throughout the

402 rest of the body (Figure $4 \mathrm{C}$ ). This is consistent with the prolonged retention of the opaque yolk

403 phenotype (Figure 1 - figure supplement 3 ) and suggests that the $m t t p^{\text {st/stl }}$ mutants are more

404 defective at secreting B-Ips from the yolk than the $m t t p^{c 655 / c 655}$ mutants.

405

406 To determine whether the mttp mutations alter the size distribution of B-lps, we performed

407 native polyacrylamide gel electrophoresis of larval homogenates expressing the LipoGlo

408 reporter. Following electrophoretic separation and chemiluminescent imaging of the gels, B-Ips

409 were classified into four different classes based on their migration distance (zero mobility, very

410 low-density lipoproteins (VLDL), intermediate-density lipoproteins (IDL), or low-density

411 lipoproteins (LDL)(Thierer et al., In Press). During development, the pattern of B-Ips in wild-type

412 embryos is initially defined by VLDL (2 dpf), but expands to include IDL and LDL by 3-4 dpf as

413 the VLDL particles produced by the YSL are lipolyzed by the body tissues (Figure 4D, Figure 4

414 - figure supplement 1, (Thierer et al., In Press)). By 5-6 dpf, the yolk is depleted and any

415 remaining small particles are degraded. In contrast, both $m t t p^{\text {st/stl }}$ and $m t t p^{c 65 / / c 655}$ embryos

416 produce very few VLDL particles (Figure 4D, $2 \mathrm{dpf}$ ), and instead, produce predominantly IDL

417 and LDL-sized particles. 


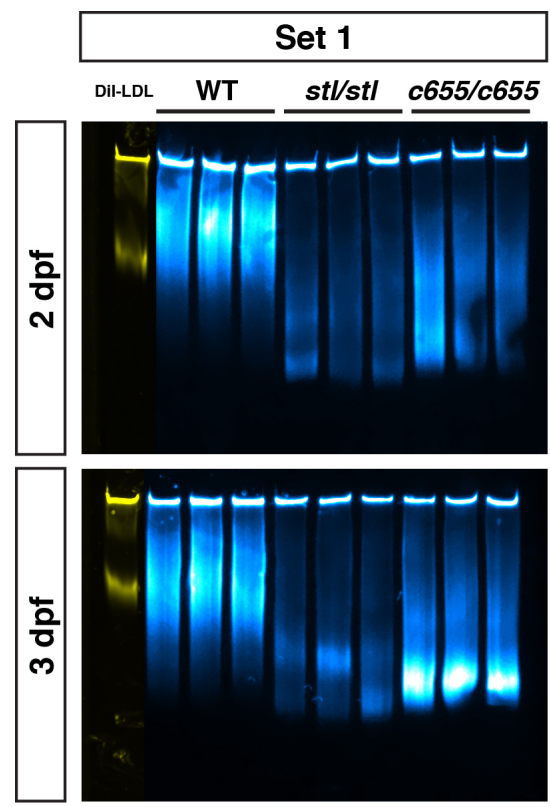

\begin{tabular}{|c|c|c|c|}
\hline \multicolumn{4}{|c|}{ Set 2} \\
\hline Dil-LDL & WT & stl/stl & c655/c655 \\
\hline
\end{tabular}

\begin{tabular}{|c|}
\hline Set 3 \\
\hline Dil-LDL WT $\quad$ stl/stl \\
\hline
\end{tabular}
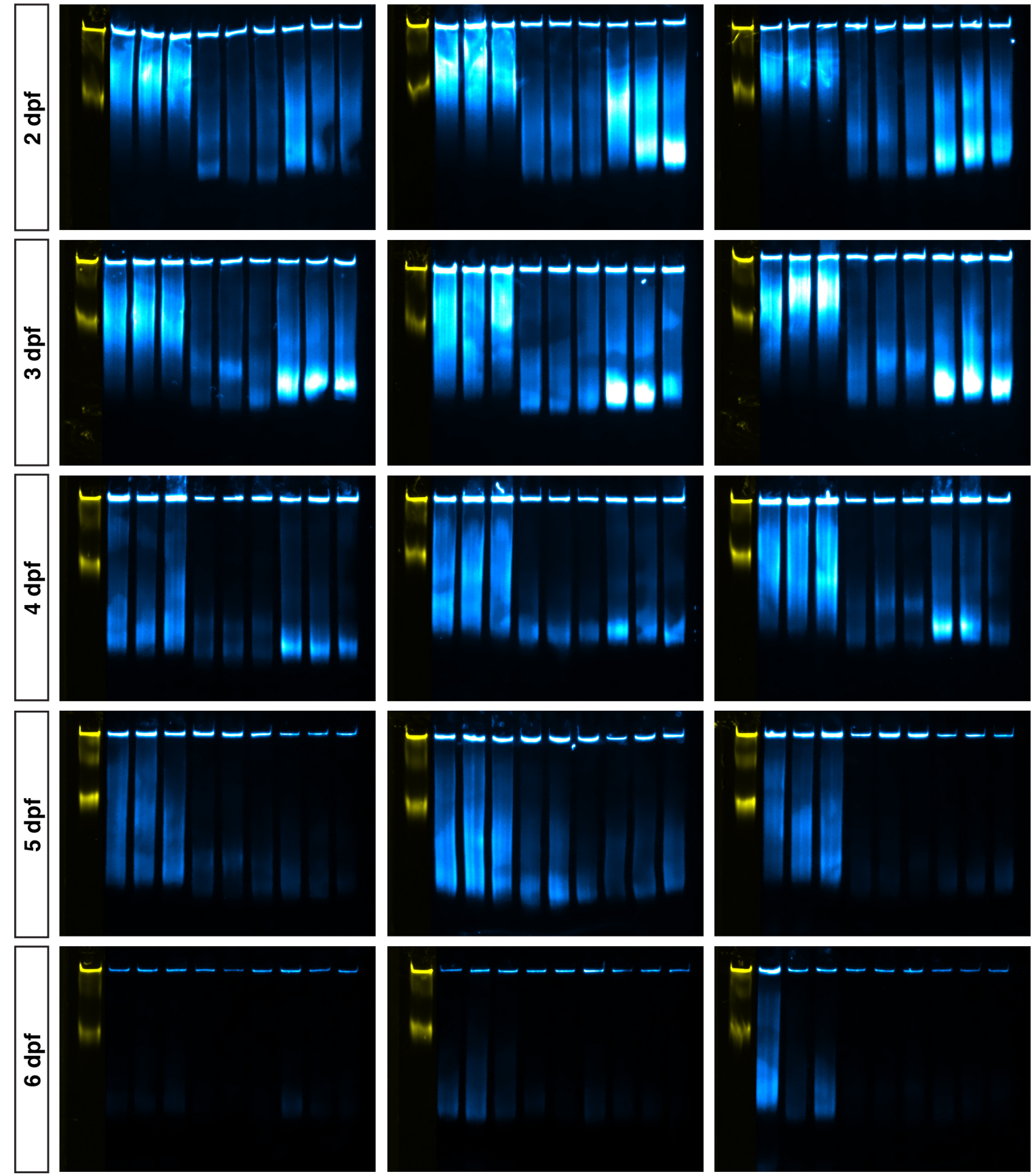

Figure 4 - figure supplement 1: LipoGlo lipoprotein gel primary data. Original gels

422 corresponding to the data in Figure 4D. Each gel shows a composite image of the fluorescent

423 Dil-LDL migration standard (yellow) and LipoGlo emission chemiluminescent exposure (blue)

424 from WT, $m t t p^{\text {st/stl }}$ and $m t t p^{c 655 / c 655}$ fish. Gels were analyzed as detailed in (Thierer et al., In

425 Press) and lipoprotein particles were binned into four classes based on migration relative to the 
426 Dil-LDL standard, including zero mobility (ZM), and three classes of serum B-Ips (VLDL, IDL 427 and LDL).

Figure 4 - figure supplement 2:

\begin{tabular}{|c|c|c|c|c|}
\hline & Zero Mobility Band & VLDL Fraction & IDL Fraction & LDL Fraction \\
\hline \multicolumn{5}{|l|}{$2 \mathrm{dpf}$} \\
\hline WT vs. stl/stl & n.s. & $p<0.0005$ & $p<0.0005$ & $p<0.0005$ \\
\hline WT vs. $c 655 / c 655$ & n.s. & $p<0.0005$ & $p<0.0005$ & $p=0.01$ \\
\hline st//st/ vs. c655/c655 & $p=0.005$ & n.s. & n.s. & \\
\hline \multicolumn{5}{|l|}{3 dpf } \\
\hline WT vs. stl/stl & $p=0.015$ & $p<0.0005$ & n.s. & $p<0.0005$ \\
\hline WT vs. $c 655 / c 655$ & n.s. & $p<0.0005$ & n.s. & $p<0.0005$ \\
\hline stl/stl vs. c655/c655 & $p=0.005$ & n.s. & n.s. & $p<0.0005$ \\
\hline \multicolumn{5}{|l|}{$4 \mathrm{dpf}$} \\
\hline WT vs. stl/stl & n.s. & $p=0.003$ & $p=0.005$ & $P<0.005$ \\
\hline WT vs. $c 655 / c 655$ & n.s. & n.s. & $p<0.0005$ & $P<0.005$ \\
\hline stl/stl vs. $c 655 / c 655$ & n.s. & n.s. & n.s. & n.s \\
\hline \multicolumn{5}{|l|}{$5 \mathrm{dpf}$} \\
\hline WT vs. stl/stl & $p<0.0005$ & $p<0.0005$ & $p<0.0005$ & n.s. \\
\hline WT vs. $c 655 / c 655$ & $p=0.025$ & $p=0.005$ & $p<0.0005$ & $p=0.005$ \\
\hline stl/stl vs. c655/c655 & n.s. & n.s. & n.s & n.s. \\
\hline \multicolumn{5}{|l|}{$6 \mathrm{dpf}$} \\
\hline WT vs. stl/stl & $p<0.0005$ & n.s. & $p<0.0005$ & n.s. \\
\hline WT vs. $c 655 / c 655$ & $p=0.03$ & n.s. & $p=0.005$ & n.s. \\
\hline stl/stl vs. c655/c655 & n.s. & n.s. & n.s. & n.s. \\
\hline
\end{tabular}

n.s. = not significant

433 Taken together, the LipoGlo data from embryos indicates that the $m t t p^{\text {st/stl }}$ and $m t t p^{c 655 / c 655}$

434 embryos are producing and secreting fewer, smaller B-Ips from the yolk, although the effect is 435 more severe in st/ mutants. Given the gross accumulation of lipid in the intestines of the adult $436 \mathrm{mtt} \mathrm{p}^{\text {st/stl }}$ mutants, compared to the $\mathrm{mttp}^{\mathrm{c655/c655}} \mathrm{fish}$ (Figure $3 \mathrm{C}$ ), we hypothesize that the $\mathrm{mttp} \mathrm{p}^{\text {st/stl }}$

437 mutants are also less effective at secreting chylomicrons from the enterocytes. To test this

438 hypothesis, we performed chemiluminescent imaging using the LipoGlo reporter in 15-dpf larvae 439 fed a chow diet for 10 days and then fasted overnight. Wild-type LipoGlo fish have abundant 440 ApoB throughout their circulation and tissues $(73.1+/-4.0 \%$ in head and trunk vs. $26.9+/-4.0 \%$

441 in viscera, mean $+/-\mathrm{SD}, \mathrm{n}=15$ fish) (Figure 4E). The $m t t p^{c 655 / c 655}$ mutation does not prevent

442 secretion of $A p o B$ to the body tissues $(73.1+/-3.7 \%$ in head and trunk vs. $26.9+/-3.7 \%$ in

443 viscera). In contrast, the $m t t p^{\text {st/stl }}$ fish have abundant LipoGlo signal in their intestine, and much

444 less in other tissues compared to WT $(41 \%+/-11 \%$ in head and trunk vs. $59+/-11 \%$ in viscera, $445 \mathrm{p}<0.001$, Kruskall-Wallis \& Dunn's Multiple Comparisons Test) (Figure 4E). In agreement, 446 staining the neutral lipid with Oil Red O indicates $m t t p^{\text {stl/stl }}$ mutants retain substantial lipid in their 
447 intestines, whereas $m t t p^{c 655 / c 655}$ mutant fish have less lipid remaining in their intestine, but do

448 accumulate some lipid in their livers (Figure 4E). This data argues that the st/ mutation severely

449 reduces B-Ip secretion, not only from the yolk, but also from the enterocytes, whereas the c655

450 mutation only mildly decreases ApoB secretion in both embryos and larvae.

The c655 mutation in zebrafish mttp disrupts the triglyceride transfer activity but not the

453 phospholipid transfer activity of the MTP complex. The dissimilar phenotypes of in vivo B-Ip

454 secretion between the stl and c655 mutations suggest that the two mutations are differentially

455 affecting MTP function. To provide molecular explanations for the phenotypes observed in the

456 different zebrafish mutants and to dissect further how each of the mutations affects MTP

457 function, we used cell and in-vitro-based assays of MTP function. COS-7 cells expressing

458 human ApoB48 were co-transfected with either an empty vector (pcDNA3), or a vector

459 containing wild-type zebrafish $m t t p$, $m t t p$-stl or $m t t p-c 655$, all with C-terminal FLAG-tags. We

460 found that $s t /$ and $c 655$ expressing COS-7 cells have significantly reduced concentrations of

461 ApoB in the conditioned media compared to wild-type Mttp expressing cells (Figure 5A). ApoB

462 levels in the media of the stl-expressing cells were similar to cells transfected with empty vector,

463 indicating background secretion levels. The concentration of ApoB48 was significantly higher in

464 the media of c655-expressing COS-7 cells compared to the st/-expressing cells and cells

465 transfected with empty vector (Figure 5A). ApoB48 concentrations in the cell lysate of cells

466 expressing st/ were significantly higher than wild-type Mttp and c655-expressing cells (Figure

467 5B). These data suggest that the st/ mutation does not support ApoB48 secretion whereas the

$468 \quad c 655$ mutation does support ApoB48 secretion, but with reduced efficacy compared to wild-type

469 Mttp. 
A

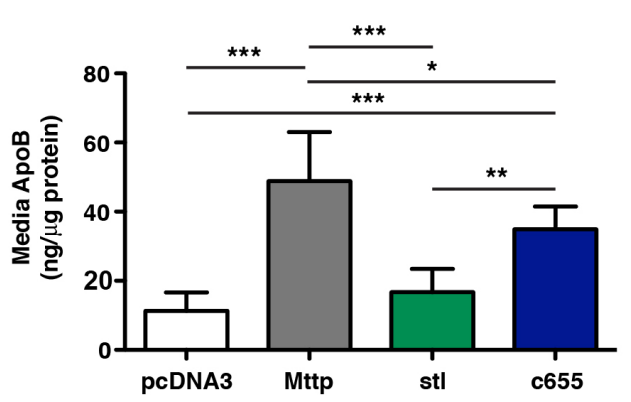

C

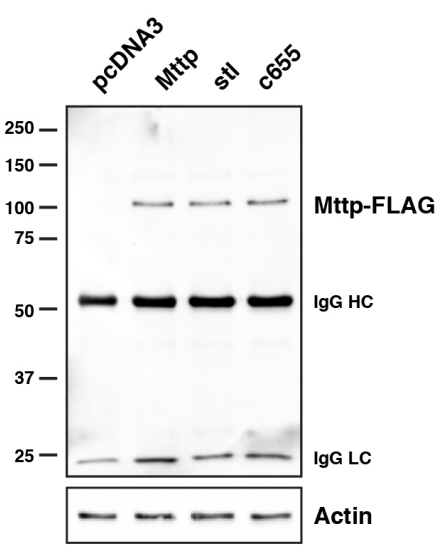

$\mathbf{E}$

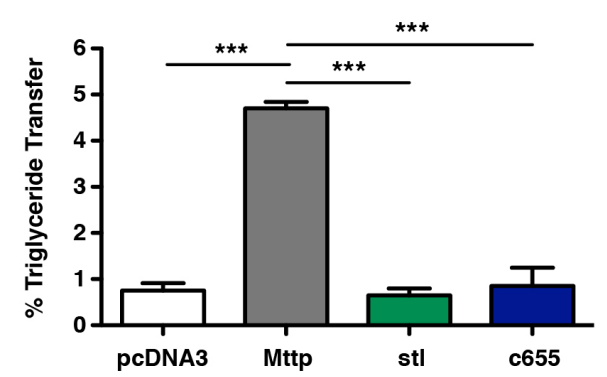

D
B
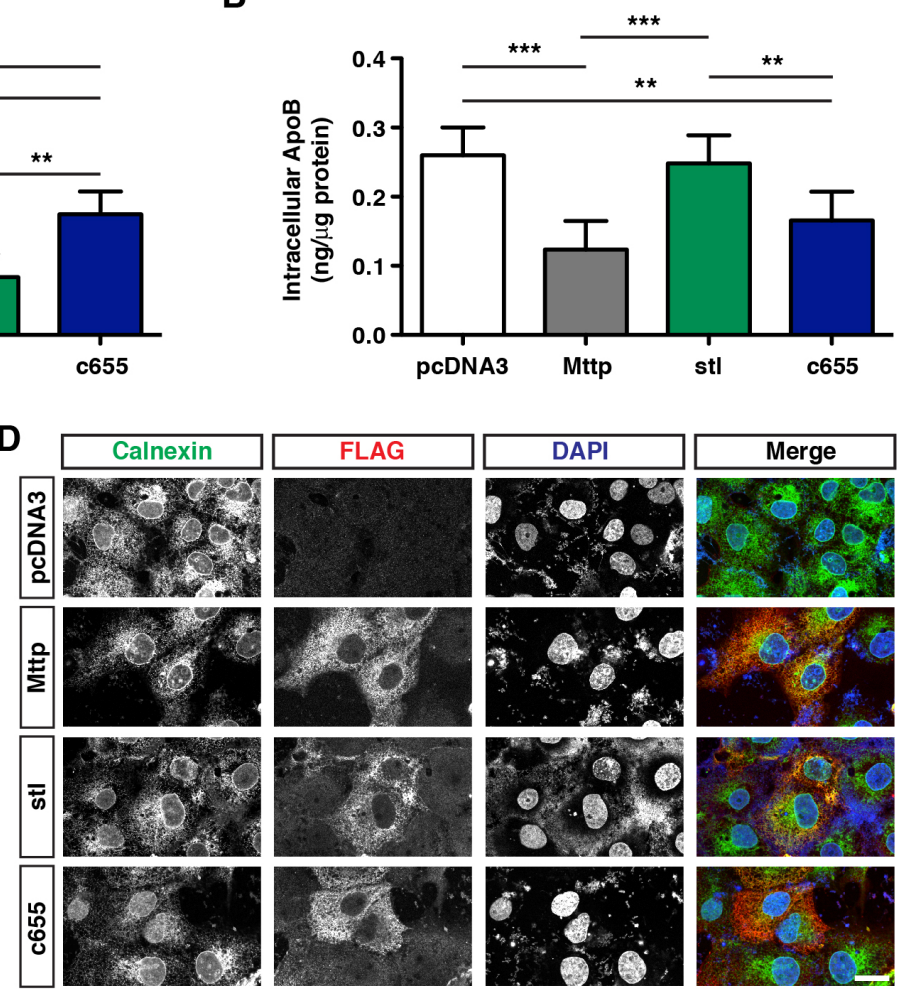

$\mathbf{F}$

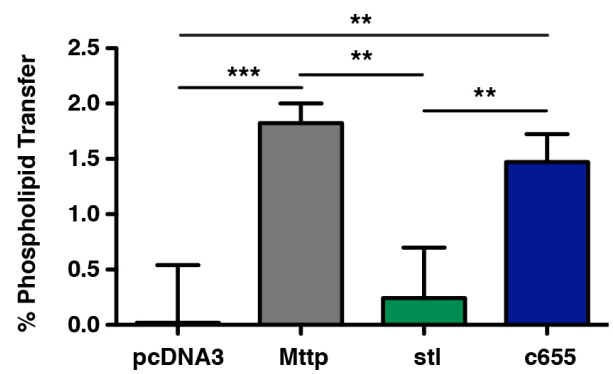

Figure 5: The $c 655$ mutation disrupts the triglyceride transfer activity, but not the phospholipid transfer activity of the zebrafish MTP complex. (A-B) COS-7 cells were first transfected with an expression vector for human apoB48 $(5 \mu \mathrm{g})$, distributed equally in 6-well plates, and subsequently transfected with plasmids expressing either wild-type zebrafish $m t t p$ FLAG, $m t t p^{s t l}$-FLAG, $m t t p^{c 655}$-FLAG, or empty vector (pcDNA3) $(3 \mu \mathrm{g})$. After $72 \mathrm{~h}$, ApoB48 was measured via ELISA in media $(A)$ or in the cell $(B)$. Data are representative of 7 independent experiments (each data point is the mean of 3 technical replicates), mean +/- SD, One-Way ANOVA with Bonferroni post-hoc tests, ${ }^{*} p<0.05$, ${ }^{* *} p<0.01,{ }^{* *} p<0.001$. (C) Cos-7 cells were transfected as described above and FLAG-tagged proteins were immunoprecipitated from cell lysates using anti-FLAG antibodies and eluted with FLAG peptides. Representative Western 
blot on eluted fractions indicates equal concentrations of the various Mttp-FLAG proteins; actin blot indicates equal loading of cell lysate. (D) Representative immunofluorescent staining using anti-FLAG (red) and anti-Calnexin (green) antibodies in COS-7 cells expressing wild-type or mutated $m t t p-F L A G$ constructs; scale $=25 \mu \mathrm{m}$. (E) COS-7 cells were transfected with plasmids expressing pcDNA3, wild-type mttp-FLAG, or mutant mttp-FLAG constructs. Cells were lysed and $60 \mu \mathrm{g}$ of protein was used to measure the \% triglyceride transfer of NBD-triolein from donor to acceptor vesicles after 45 minutes; $n=3$ (each $n$ is the mean of three technical replicates from independent experiments), mean +/- SD, One-way ANOVA with Bonferroni post-hoc tests, ${ }^{* * *} p<0.001$. (F) Wild-type and mutant Mttp proteins were purified using anti-FLAG antibodies and used to measure the \% transfer of NBD-labeled phosphoethanolamine triethylammonium from donor to acceptor vesicles after 180 minutes; $n=3$ (each $n$ is the mean of 3 technical replicates from independent experiments), mean $+/-$ SD, randomized block ANOVA with Bonferroni post-hoc tests, ${ }^{\star * *} p<0.001$.

To eliminate the possibility that the st/ mutation did not support ApoB48 secretion due to low expression, Mttp, stl, and c655 were precipitated using anti-FLAG antibodies from cell lysates and were subjected to Western blot analysis. We found that there was no difference in the expression of wild-type Mttp and Mttp mutants in COS-7 cells (Figure 5C). Another reason for

502 the st/ mutation to be deficient in supporting ApoB48 secretion could be due to protein mis503 localization. To check this, we immunostained Mttp-expressing COS-7 cells with anti-Calnexin 504 and anti-FLAG antibodies. Confocal imaging shows that the wild-type Mttp and both mutant 505 proteins were properly localized in the endoplasmic reticulum (Figure 5D). Additionally, the 506 percentage of cells expressing the FLAG-tagged proteins were similar among all groups (Mttp507 FLAG 37\%, Mttp-st/31\%, Mttp-c655 41\% transfection efficiency). These studies suggest that 508 stl and c655 mutant Mttp proteins are expressed to similar levels and are in the ER, where 509 lipoprotein assembly occurs.

511 Next, we hypothesized that the st/ mutant protein may not support ApoB48 secretion because it 512 might be defective in lipid transfer activity. Triglyceride transfer assays performed using cell

513 lysates showed that both of the mutant forms of Mttp (st/ and c655) have significantly decreased 514 triglyceride transfer activity compared to wild-type zebrafish Mttp (Figure 5E, Figure 5 - figure 515 supplement 1a). The st/ mutant form was also found to be defective in phospholipid transfer 
activity compared to wild-type (Figure 5F, Figure 5 - figure supplement $1 \mathrm{~b}$ ). Contrary to this, the $c 655$ mutant form had similar phospholipid transfer activity to wild-type Mttp (Figure 5F,

518 Figure 5 - figure supplement $1 \mathrm{~b}$ ). These data suggest that the $c 655$ mutation in mttp impairs

519 triglyceride transfer, but not phospholipid transfer activity. In contrast, the stl mutation is defective in both transfer activities. These differences in lipid transfer activities between the stl and c655 mutations provide a biochemical explanation for their differential abilities to support ApoB48 secretion and the different phenotypes observed in the fish.

A

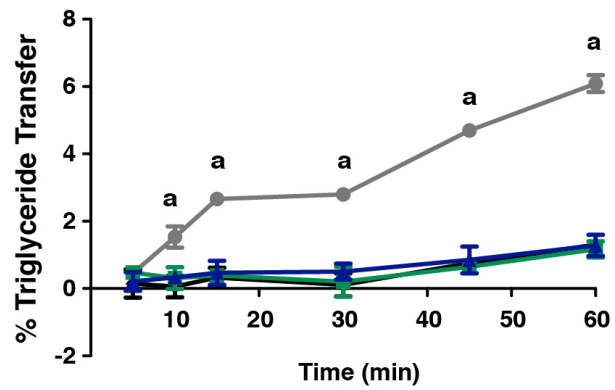

a; pcDNA3 vs. all others, $p<0.001$

b; zmttp vs. stl, p<0.05

c; pcDNA3 \& stl vs. zmttp \& c655, p<0.01

d; pcDNA3 vs. zmttp \& c655, p<0.001

e; stl vs. zmttp, $p<0.001$

f: stl vs. $c 655, p<0.01$
B

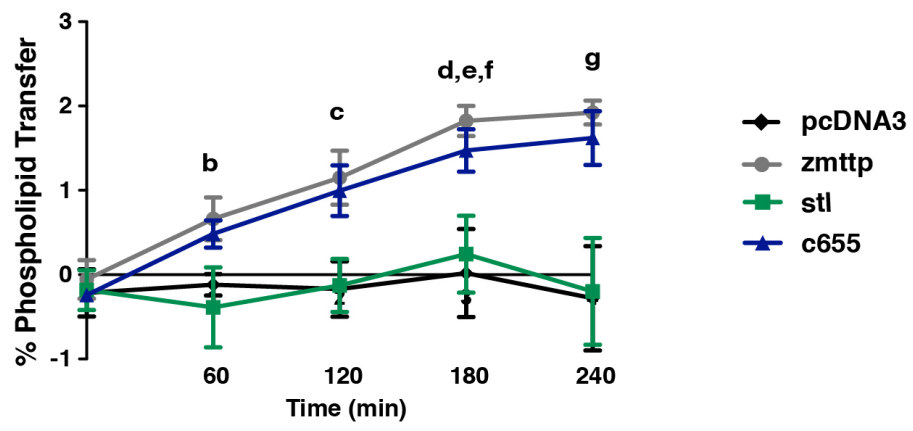

Figure 5 - figure supplement 1: Triglyceride and phospholipid transfer assay timecourse data. Measurements for triglyceride (A) and phospholipid transfer (B) by zebrafish $m t t p-F L A G$ and mutants over a time-course. The single time-points depicted in the bar graphs of figures $5 E \& \mathrm{~F}$, correspond to the $45 \mathrm{~min} \& 180 \mathrm{~min}$ (triglyceride and phospholipid transfer, respectively) time-points in the curves shown. For both, $n=3$ (each $n$ is the mean of 3 technical replicates from independent experiments), mean +/- SD, Repeated Measures ANOVA with Bonferroni post-hoc tests, significance as noted in figure.

\section{An orthologous c655 mutation in human MTTP (G865V) also disrupts the triglyceride} transfer activity but not phospholipid transfer activity. The M subunit of MTP shares homology with lipovitellin, a lipid transfer protein in the yolk of oviparous animals (Banaszak et al., 1991). Homology modeling with the crystal structure of lamprey lipovitellin (PDB ID: 
1LSH)(Anderson et al., 1998; Raag et al., 1988; Thompson and Banaszak, 2002) predicts three

541 major structural domains in human MTTP: an N-terminal beta-barrel, a middle alpha-helical

542 domain, and a C-terminal domain composed of two beta-sheets that form a hydrophobic lipid-

543 binding cavity (Hussain et al., 2003a; Mann et al., 1999; Read et al., 2000). The amino acid

544 sequence of the zebrafish Mttp is $54 \%$ identical ( $72 \%$ similar) to that of human MTTP and the

545 predicted secondary and tertiary structures are highly conserved (Figure 6A).

547 The leucine residue mutated in the st/ mutant fish (L475P) is conserved in human MTTP (L477)

548 and lies within a highly conserved stretch of amino acids located in the middle alpha-helical

549 domain of MTTP (Figure 6A). Analysis of mutations in patients with Abetalipoproteinemia has

550 shown that missense mutations in the alpha-helical domain often prevent lipid transfer activity

551 and result in loss of $A p o B$ secretion (Reviewed in (Walsh and Hussain, 2017)). Therefore, it is

552 likely that the stI L475P mutation alters the structure of the lipid-binding cavity in a similar

553 manner to these patient mutations.

555 The glycine residue mutated in the C-terminus of $c 655$ mutants (G863V) is also conserved in

556 the human sequence (G865) (Figure 6A). Unfortunately, it is unknown where the G865 residue

557 is located in relation to the lipid-binding cavity because the C-terminal sequence of MTTP

558 diverges from that of lipovitellin, so it is not modeled in the predicted MTTP structure. However,

559 because the predicted tertiary structure of zebrafish Mttp is very similar to that of human MTTP

560 and the sequence in the C-terminus is highly conserved (Figure 6A), we hypothesized that

561 introduction of the $c 655$ mutation into the human MTTP protein would also cause loss of

562 triglyceride transfer, but retention of phospholipid transfer activity. To test this hypothesis, we

563 performed site-directed mutagenesis on the human MTTP-FLAG (hMTTP) plasmid and

564 assessed the function of the MTTP (G865V) mutant protein. 
A

Human 460-GLEKAEKKEDTRMYLLALKNALLPEGIPSLLKYAEA-495 Zebrafish 458-GPDSTQEESEVQMYLLALKNALLPEGIPVLAKYAES-493

$$
\text { Kstl (L475P) }
$$

Human 850-TGRGYVSQKRKESVLAGCEFPLHQENSEMCKVVFAPQPDSTSSGWF* Zebrafish 848-TGQMFSRKRSRDQVVPGSEFPLHQENSNMCKKVFEPAW*

C655(G865V)

Human MTTP (Predicted)

Zebrafish Mttp (Predicted)

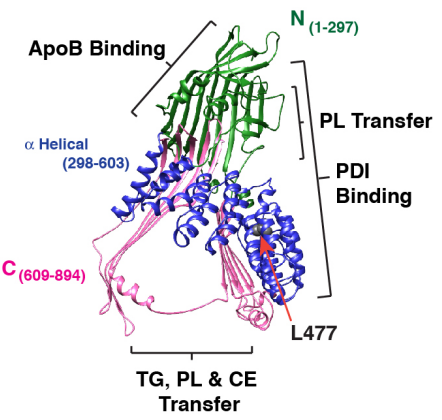

$\mathbf{E}$

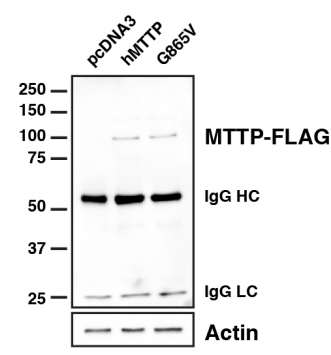

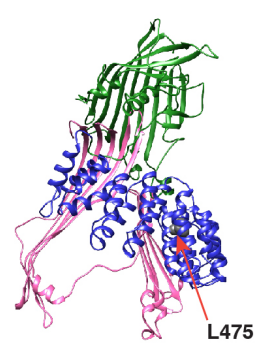

$\mathbf{F}$

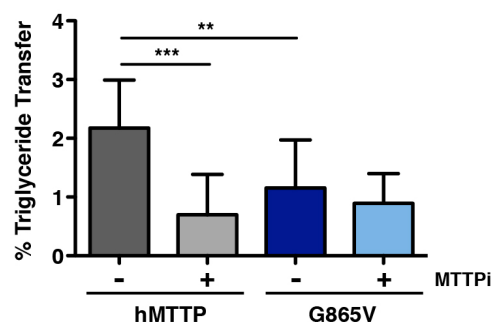

D
C
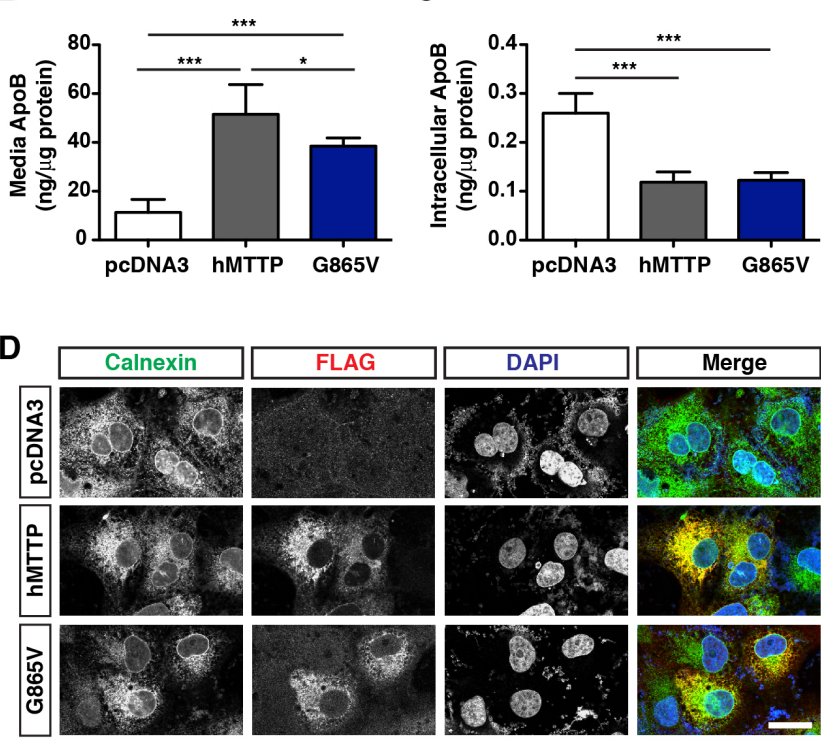

G

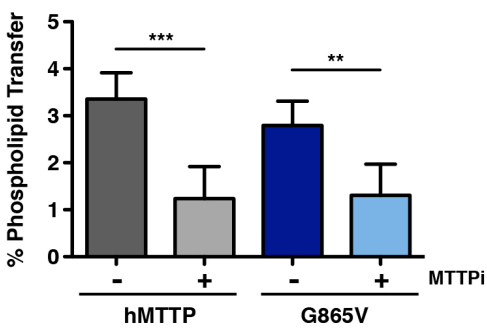

Figure 6: The $c 655$ mutation in human MTTP also disrupts the triglyceride transfer activity, but not the phospholipid transfer activity of the MTP complex. (A) Alignment of human and zebrafish Mttp amino acid sequences surrounding the stl or c655 mutations. Ribbon diagrams of the predicted tertiary structures of human MTTP and zebrafish Mttp modeled using Phyre2 (Kelley et al., 2015) based on the lamprey lipovitellin structure (Anderson et al., 1998). The predicted phospholipid transfer sites in the $\mathrm{N}$-terminal region (green) and the C-terminal region (pink, composed of two beta-sheets that form a hydrophobic lipid-binding pocket) are labeled. The location of the conserved human residue (L477) corresponding to the zebrafish stl (L475P) mutation is noted. The C-terminal region containing the c655 mutation diverges from the lipovitellin structure and is not reliably modeled. (B,C) The c655 mutation (G865V) was introduced into the human MTTP-FLAG plasmid. COS-7 cells were co-transfected with human ApoB48 and either wild-type human MTTP-FLAG, MTTP(G865V)-FLAG or empty pcDNA3 plasmids. After $72 \mathrm{~h}$, apoB48 was measured via ELISA in media (B) or in the cell (C). Data are representative of 7 independent experiments (each data point is the mean of 3 technical replicates), pcDNA3 control data is re-graphed from figure 5A \& 5B (data for 5A, 5B, $6 \mathrm{~B} \& 6 \mathrm{C}$ 
were generated together); mean +/- SD, One-Way ANOVA with Bonferroni post-hoc tests, * $p<0.05,{ }^{* * *} p<0.001$. (D) Immunofluoresence in COS-7 cells expressing wild-type human

585 MTTP-FLAG or human MTTP(G865V)-FLAG using anti-FLAG (red) and anti-Calnexin (green) antibodies; scale $=25 \mu \mathrm{m}$. (E) COS-7 cells were transfected and FLAG-tagged human MTTP proteins were immunoprecipitated from cell lysates using anti-FLAG antibodies and eluted with FLAG peptides. Representative Western blot on eluted fractions indicates equal concentrations of the various MTTP-FLAG proteins; actin blot indicates equal loading of cell lysate. (F) COS-7 cells were transfected with plasmids expressing human wild-type or MTTP(G865V)-FLAG constructs. Cells were lysed and $60 \mu \mathrm{g}$ of protein was used to measure triglyceride transfer

592 activity in the presence or absence of the MTTP inhibitor lomitapide $(1 \mu \mathrm{M})(\%$ after 45 minutes);

$593 n=9$ (3 measurements from each of 3 independent experiments), mean +/- SD, One-way

594 ANOVA with Bonferroni post-hoc tests, ${ }^{\star *} p<0.01,{ }^{* \star *} p<0.001$ ). (G) Wild-type and mutant MTTP

595 proteins were purified using anti-FLAG antibodies and used to measure phospholipid transfer in 596 the presence or absence of lomitapide $(1 \mu \mathrm{M})$ (180 minutes); $\mathrm{n}=9$ (3 measurements from each 597 of 3 independent experiments), mean +/- SD, randomized block ANOVA with Bonferroni post598 hoc tests, ${ }^{\star *} p<0.01,{ }^{* \star *} p<0.001$. COS-7 cells expressing the G865V mutant protein secreted slightly lower amounts ( $75 \%)$ of ApoB48 into the media compared to wild-type hMTTP, but this was significantly higher than the

603 background levels seen in cells transfected with the empty vector (pcDNA3) (Figure 6B).

604 ApoB48 concentrations in the cell lysate of cells expressing hMTTP and G865V mutant proteins 605 were similar (Figure 6C). These data suggest that the G865V mutation supports ApoB48 606 secretion albeit at lower efficiency. Attempts were made to explain the mechanisms for lower 607 efficiency in supporting ApoB secretion. Wild-type hMTTP-FLAG and G865V-FLAG were both 608 localized to the ER (Fig. 6D), transfection efficiency was similar (36\% hMTTP, 35\% G865V), 609 and the proteins were immunoprecipitated at similar levels from cell lysates (Figure 6E).

610 Triglyceride transfer assays with cell lysates of COS-7 cells expressing hMTTP-FLAG and 611 G865V-FLAG plasmids showed that the mutant G865V protein has significantly decreased $612(\sim 53 \%)$ triglyceride transfer activity compared to wild-type hMTTP, comparable to the activity 613 level of the WT hMTTP in the presence the MTTP inhibitor lomitapide ( $1 \mu \mathrm{M})$ (Figure $6 \mathrm{~F})$. In 614 contrast, the phospholipid transfer activity of hMTTP and G865V were not significantly different, 
615 and the activity of both alleles were inhibited to equal extent by lomitapide (Figure 6G). These

616 data suggest that the c655 mutation in both the zebrafish Mttp (G863V) and human MTTP

617 protein (G865V) results in significant loss of triglyceride transfer activity, but has no effect on

618 phospholipid transfer activity.

\section{DISCUSSION}

624 The characterization of the zebrafish mttp c655 mutation provides the first evidence that the

625 triglyceride and phospholipid transfer functions of a vertebrate microsomal triglyceride transfer

626 protein can be dissociated and identifies the putative region responsible for triglyceride transfer

627 activity. Previous sequence comparisons of invertebrate and vertebrate orthologues of MTTP

628 strongly suggested that acquisition of triglyceride transfer activity during evolution was the result

629 of many changes in the lipid-binding cavity (Rava and Hussain, 2007), so it was unexpected that

630 one missense mutation in the C-terminus selectively eliminated triglyceride transfer activity.

631 Additionally, all of the characterized missense mutations from patients with

632 Abetalipoproteinemia cause MTP to be either absent or deficient in both phospholipid and

633 triglyceride transfer activities (Berthier et al., 2004; Di Filippo et al., 2012; Di Leo et al., 2005;

634 Khatun et al., 2013; Miller et al., 2014; Narcisi et al., 1995; Rehberg et al., 1996; Ricci et al.,

635 1995; Walsh et al., 2016; Walsh and Hussain, 2017; Walsh et al., 2015; Wang and Hegele,

636 2000).

638 While we do not know where the C-terminus is located in relation to the lipid-binding cavity

639 (Figure 6A), one of the Abetalipoproteinemia mutations (G865X) results in a C-terminal

640 truncation of 30 amino acids that prevents binding to PDI, thus resulting in loss of MTP protein

641 (Ricci et al., 1995). Subsequent analysis of constructs in vitro indicated that deletion of the last

64220 amino acids ( $\Delta 20, \mathrm{~S} 875 \mathrm{X})$, or mutating the cysteine at position 878 (C878S), reduced the

643 expression of the protein to $<15 \%$ of wild-type MTTP levels and abolished triglyceride transfer

644 activity (Narcisi et al., 1995). This cysteine residue is conserved in MTTP orthologues from

645 human to $C$. elegans, suggesting that it forms a disulfide bond essential for the tertiary structure

646 of the protein. The authors argue that the residual protein produced must be binding to PDI,

647 otherwise, no protein would be detected; however, it is unclear whether either of these mutated

648 proteins are still capable of transferring phospholipid (Narcisi et al., 1995). In contrast to these 
mutations, the mutation of glycine to valine at position 865 in human MTTP (or 863 in zebrafish mttp) does not reduce protein expression or alter the localization of MTTP in the ER of COS-7 cells (Figure 5D,6D), indicating that the mutation does not interfere with PDI binding. This suggests that the proposed disulfide bond is intact and that the G865V mutation may be more directly affecting lipid transfer. Our data show that this mutation lacks the ability to transfer

654 triglycerides in vitro, but it is unclear whether the mutation also prevents binding of triglyceride in 655 the lipid-binding cavity. Perhaps binding can still occur, which would be consistent with the 656 evolutionary data, and maybe the C-terminus is necessary for transfer activity. Analyses of 657 Drosophila, zebrafish, or human MTTP chimeric proteins may help test this hypothesis in the 658 future. Additionally, in order to better understand how the c655 mutation specifically inhibits triglyceride transfer, a crystal structure including the C-terminus will likely be necessary.

The production of B-Ips in the ER of the intestine and liver is thought to occur in two steps. In

662 the first step, MTP transfers lipids to ApoB as it is translated to form small primordial particles.

663 In the second step, it has been suggested that fusion of ApoB-free lipid droplets in the lumen of 664 the ER fuse to expand the lipoprotein core ("core expansion") (Alexander et al., 1976; Boren et 665 al., 1994; Hamilton et al., 1998; Wang et al., 1997). There is evidence to suggest that MTP is 666 also responsible for producing these ER-lumenal lipid droplets (Kulinski et al., 2002). Using our 667 LipoGlo assays, we have shown that the c655 mutant fish produce small, homogenous, 668 particles, whereas the wild-type embryos form VLDL-sized lipoproteins in the YSL at 2-3 dpf 669 (Figure 4D). We have made similar observations in liver-specific Mttp KO mice expressing 670 Drosophila Mttp, which has robust phospholipid transfer activity, but is deficient in triglyceride 671 transfer (Khatun et al., 2012). Expression of fly Mttp resulted only in production of small B-Ips, 672 but human MTTP rescued the particle size (Khatun et al., 2012). Therefore, the phospholipid 673 transfer activity of MTP may be crucial in the generation of the small homogenous particles 674 representative of the first step of lipoprotein assembly, whereas triglyceride transfer might be 675 primarily responsible for core expansion.

Although the effect of the $c 655$ mutation on the molecular function of the protein was

678 unexpected, the lack of intestinal or hepatic steatosis is also consistent with our previous data 679 with Drosophila Mttp expression in the livers of liver-specific Mttp-null mice. The phospholipid680 rich high-density B-Ips produced by the fly Mttp in hepatocytes partially restore plasma lipid 681 levels and reduce liver steatosis (Khatun et al., 2012). Similarly, transfer of phospholipid and 
682 production of small B-Ips in the 6655 mutant fish is not only sufficient for moving lipid from the

683 liver, but is also capable of moving enough dietary lipid and fat-soluble vitamins from the

684 intestine to prevent intestinal steatosis and support normal growth (Figure 3,4). Furthermore,

685 retention of phospholipid transfer may also improve the health of the fish in ways that are

686 independent of lipoprotein production. For example, MTP-dependent phospholipid transfer has

687 been shown to be important for biogenesis and cell surface expression of CD1d and possibly

688 other lipid-antigen-presenting molecules (Dougan et al., 2005).

690 In contrast to the health and viability of $c 655$ mutants, the st/ mutant fish that survive have gross

691 lipid accumulation in their intestine and severe growth defects (Figure 3,4), which is reminiscent

692 of patients with Abetalipoproteinemia. The mutant protein localizes normally to the ER, but is

693 deficient in triglyceride transfer, phospholipid transfer, and ApoB secretion (Figure 5). In the

694 predicted model of the tertiary structure of Mttp, this mutation is located in the middle alpha-

695 helical domain, not facing the lipid-binding cavity. The location and characterization of this

696 mutation is very similar to that of the described $\mathrm{Y} 528 \mathrm{H}$ and S590I Abetalipoproteinemia

697 mutations, and it is likely that all three mutations prevent lipid transfer by altering the tertiary

698 structure of the M subunit (Khatun et al., 2013; Miller et al., 2014).

Counter to the original characterization of stl mutants (Avraham-Davidi et al., 2012), we show that the st/ mutants can survive to adulthood, especially when they are not competing with siblings for resources. We hypothesize that during the maintenance of this mutant line since its original characterization, a modifier has been eliminated that, when present in the stl

704 background, was incompatible with life. In support of this hypothesis, the excessive sprouting 705 angiogenesis defect for which the stalactite mutation was named (Avraham-Davidi et al., 2012), 706 was also not as severe as originally described (Supplementary Figure S1). Whether the 707 proposed modifier directly affects the secretion of B-lps, or some other aspect of development, 708 is currently unclear. While the st/ mutation severely decreases B-Ip number and particle size 709 (Figure 4D), some ApoB is still noted in the body of the $m t t p^{\text {stlstl }}$ embryos and larvae (Figure 4C,

$7104 \mathrm{E})$. This suggests that there is enough transport of necessary lipids and fat-soluble vitamins by 711 the small numbers of B-Ips produced by the YSL and intestine to support development. 
A

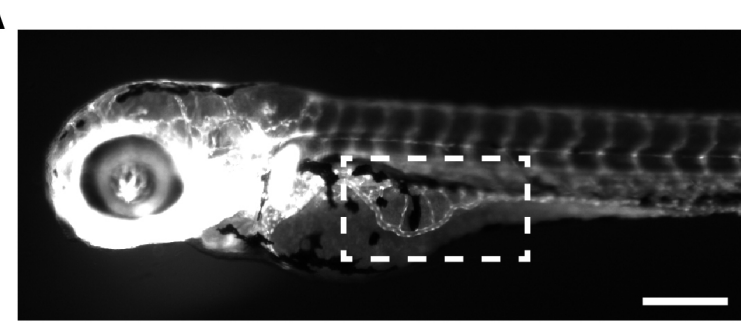

C

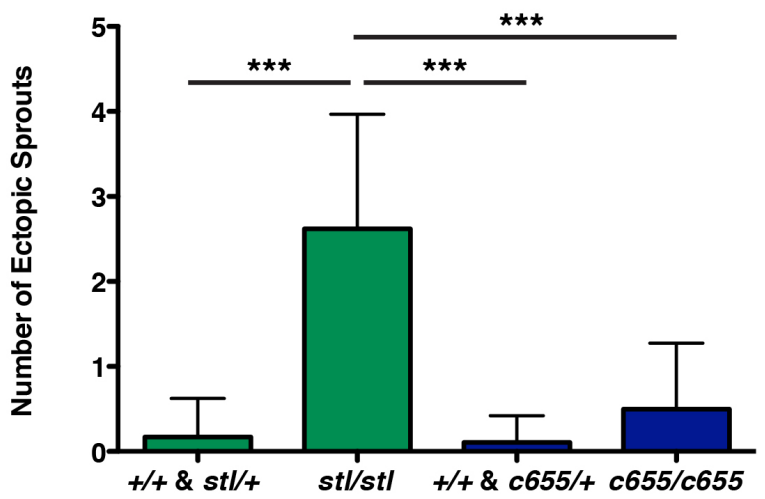

B
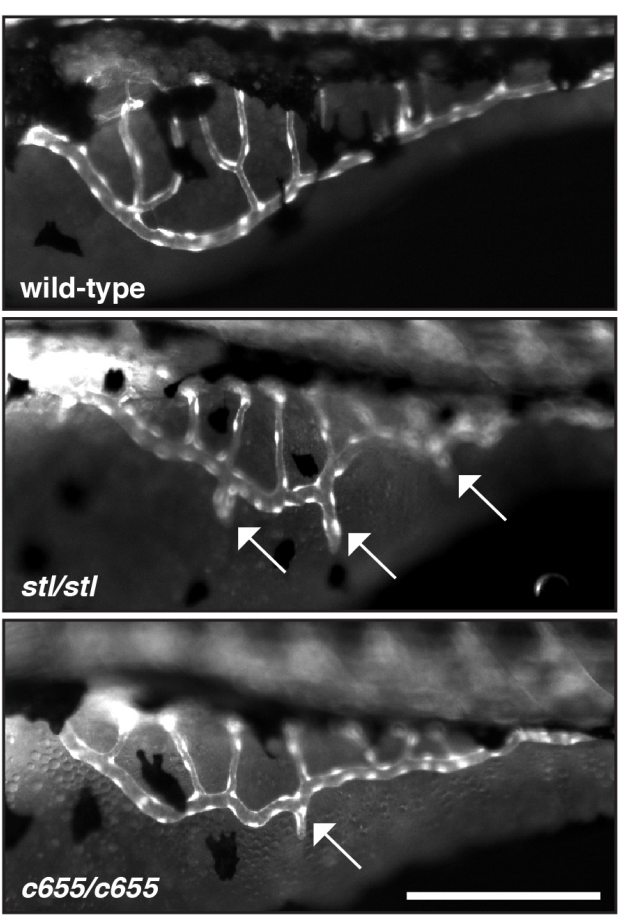

713

714

715

716

Figure S1: c655/c655 embryos exhibit fewer ectopic angiogenic segments extending

\section{from the subintestinal vessels than stl/stl embryos. (A) The developing vasculature is} visualized in the $T g(f l i: e G F P)^{y 1}$ transgenic zebrafish line (Lawson and Weinstein, 2002). The subintestinal vessels (boxed region) grow bilaterally onto the dorsolateral surface of the yolk sac. Scale $=200 \mu \mathrm{M}$. (B) Representative wide-field images of $T g(f l i: e G F P)^{y 1}$ in wild-type, $m t t p^{\text {stl/stl }}$ or $m t t p^{c 655 / c 655}$ embryos at $3.5 \mathrm{dpf}$. Ectopic sprouts extending ventrally from the

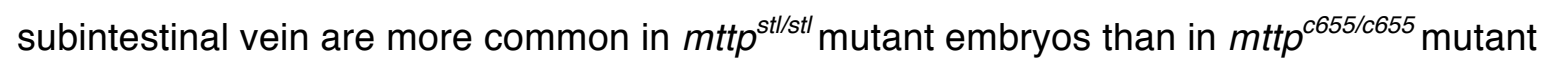
embryos. Scale $=200 \mu \mathrm{M}$. (C) Quantification of the average number of ectopic sprouts in $m t t p$ mutants and siblings on $3.5 \mathrm{dpf}$. Results represent pooled data from 3 independent experiments, $\mathrm{n}=$ 28-36 total embryos/genotype group; mean +/- SD, Kruskall-Wallis with Dunn's Multiple Comparison test, ${ }^{* *} p<0.001$.

The c655 mutants were initially identified due to the abnormal opaque appearance of their yolks, which is also a component of the st/ mutant phenotype (Figure 1). We show that this opacity is due to the abnormal accumulation of lipid droplets in the yolk syncytial layer (Figure 2). As the lipids are liberated from the yolk, they are rapidly re-esterified to triglyceride and phospholipid and packaged into B-Ips in the endoplasmic reticulum of the YSL. Because the mttp mutant fish are defective at producing (st/) and/or expanding (c655) B-Ips, the re-esterified lipids are 
733 packaged instead into cytoplasmic lipid droplets. In contrast, cytoplasmic lipid droplets are only

734 very rarely noted in the YSL of wild-type embryos, suggesting that the rate of yolk lipid break-

735 down and the rate of B-Ip production and secretion from the YSL are exquisitely coupled. We

736 also noted that the size range of the cytoplasmic lipid droplets in the YSL of the c655 mutants

737 was much larger than in the st/ mutants, with some LDs reaching $180 \mu \mathrm{m}^{2}$. As the surface area-

738 to-volume ratio decreases with increasing size spheres, the larger lipid droplets store more

739 neutral lipid relative to the surface of the phospholipid coat. Because the c655 mutants can

740 transfer phospholipid to ApoB and secrete greater numbers of small dense B-Ips than stl

741 mutants, we hypothesize the retained neutral lipid is stored in larger lipid droplets because the

742 phospholipid is being secreted and less available to coat lipid droplets. However, it is unclear

743 whether the larger LDs form due to local production of triglycerides and cholesterol esters

744 directly on their surface, or due to lipid droplet fusion, or both mechanisms (reviewed in

745 (Olzmann and Carvalho, 2019; Walther et al., 2017)). The differences in the concentration and

746 size of LDs between the mutants may result in differential effects on the degree of light

747 scattering, which could explain the differences in opacity noted between mutants (Figure 1 -

748 figure supplement 3).

None of the missense mutations identified in patients with Abetalipoproteinemia have been found to dissociate the lipid transfer activities of MTP (reviewed in (Walsh and Hussain, 2017)).

752 However, given that the adult c655 mutant zebrafish are indistinguishable from wild-type

753 siblings, it is entirely possible that humans carrying a missense mutation that results in retention

754 of phospholipid transfer exist in the population. While these people would likely have low

755 plasma triglycerides, we would expect transport of fat-soluble vitamins to be normal. A thorough

756 search of publicly available large human GWAS databases (Global BioBank Engine, T2D

757 Knowledge Portal, GTEx Portal) did not reveal any coding variants near G865 other than the

758 G865X mutation discussed above. However, since one copy of wild-type MTTP is sufficient to

759 prevent fat malabsorption when faced with an oral fat load (Di Filippo et al., 2019), individuals

760 heterozygous for a mutation similar to $c 655$ may not present with any changes in plasma lipid

761 profiles.

762

763 Abnormally elevated levels of ApoB-containing lipoproteins and remnants promote

764 atherosclerosis, the leading cause of death in the United States (CDC, 2018). Inhibition of MTP

765 has long been considered a possible therapeutic target for lowering disease risk by inhibiting the 
766 production of VLDL and chylomicrons (Jamil et al., 1996; Wetterau et al., 1998)(for review see

767 (Hussain and Bakillah, 2008; Walsh and Hussain, 2017)). Currently, the only MTP inhibitor

768 approved for use in patients is lomitapide (Juxtapid $\AA$ ), which inhibits triglyceride and

769 phospholipid transfer and reduces ApoB secretion (Robl et al., 2001)(Figure 6F,G). While this drug effectively reduces LDL cholesterol, total cholesterol, and plasma ApoB levels, it is only approved for patients with homozygous familial hypercholesterolemia, whose plasma

772 cholesterol and triglyceride levels are up to four times the normal levels resulting in premature

773 cardiovascular disease (Cuchel et al., 2014; Cuchel et al., 2013; FDA, 2012). While lomitapide

774 effectively lowers circulating lipid levels and reduces cardiovascular disease risk in these

775 patients, side effects include fat accumulation in the liver and adverse gastrointestinal events

776 including reflux, indigestion, abdominal pain, constipation, and diarrhea (Blom et al., 2017;

777 Cuchel et al., 2007; Cuchel et al., 2013).

The lack of intestinal and hepatic steatosis in the c655 mutant fish suggests that an MTP inhibitor that selectively targets triglyceride transfer activity could potentially lower plasma lipids while preventing these gastrointestinal and liver side effects. This would not only improve the

782 quality of life for patients currently taking lomitapide, but may also expand MTP inhibitor use to

783 patients other than those with familial hypercholesterolemia. While one of the original MTP

784 inhibitors discovered, BMS-200150, was very effective at inhibiting triglyceride transfer, but less

785 effective ( 30\%) at inhibiting phospholipid transfer in vitro (Jamil et al., 1996), later studies on

786 purified MTP protein indicated the compound inhibits transfer of both lipid classes (Rava et al.,

787 2006) and that it was not effective in animal models (Wetterau et al., 1998). Now that we

788 appreciate that the triglyceride and phospholipid transfer functions of MTTP can be dissociated,

789 we argue that it may be worth re-evaluating the phospholipid transfer activity of any previously

790 identified compounds that inhibited triglyceride transfer activity of MTP, but failed to inhibit ApoB

791 secretion in vitro. Perhaps new compounds could be specifically designed to target the C-

792 terminal region of MTTP, although this will likely require a crystal structure of the MTP complex.

794 However, before a selective triglyceride transfer inhibitor could be considered as a therapeutic

795 for a wide range of patients with hyperlipidemia, it will be important to determine whether the

796 small lipoprotein particles produced by selective inhibition of triglyceride transfer are not

797 atherogenic. Small dense LDL has been found to have greater potential for causing

798 atherosclerosis than larger LDL sub-fractions and is a better predictor of cardiovascular disease 
than total LDL-cholesterol (Austin et al., 1988; Bjornheden et al., 1996; Ivanova et al., 2017). In future work, we want to evaluate whether the $m t t p^{c 655 / 6655}$ mutants are protected from atherosclerosis because they produce fewer B-Ips or are at higher risk because the B-Ip particles secreted are small and dense.

804 In conclusion, the unexpected discovery of the c655 missense mutation in mttp has provided 805 novel insight into the structure-function relationship of MTP, underlining the importance of

806 forward-genetic screening approaches to reveal aspects of biology that may otherwise be

807 missed. Our work provides the first evidence that the triglyceride and phospholipid transfer

808 functions of vertebrate MTP can be separated and that selective retention of phospholipid

809 transfer is sufficient for dietary fat absorption and normal growth. These results argue that

810 selective pharmacologic inhibition of triglyceride transfer may be a feasible therapeutic

811 approach to treat disorders of lipid metabolism.

\section{Acknowledgments}

814 We gratefully acknowledge Michael Sepanski for electron microscopy, Andrew Rock and

815 Carmen Tull for fish husbandry, Matthew Bray for assistance using R, Amy Kowalski for

816 synthesis of the pDESTToI2pA2-CMV: eGFP-CAAX plasmid, and Jennifer Anderson for help

817 editing the manuscript. We would also like to especially thank Philip Ingham, who provided the

818 kif7 mutant strain in which we identified the c655 mutation. This work was supported by NIH

819 grants R01 DK093399 (Farber, PI; Busch-Nentwich, Co-PI), R01 GM63904 (The Zebrafish

820 Functional Genomics Consortium; Ekker, PI, Farber, Co-PI), HL-137202-01A1 (Hussain, PI),

821 R56 DK046900-17A1 (Hussain, PI) and F32DK109592 to M.H.W., as well as G. Harold \& Leila

822 Y. Mathers Foundation (Farber, PI), VA Merit Award BX004113-01A1 (Hussain, PI), AHA

823 Postdoctoral Fellowship 19POST34410063 to S.R., and the Wellcome Trust [098051 and

824 206194] to E. Busch-Nentwich.

Declaration of Interests

827 Authors declare no competing interests.

\section{Supplementary Files}

831 Supplementary File 1: Single nucleotide variants present in c655 mutant embryos. 


\section{METHODS \& MATERIALS}

835

836

837

838

839

840

841

842

843

844

845

846

847

\section{Zebrafish husbandry and maintenance}

Adult zebrafish were maintained at $27^{\circ} \mathrm{C}$ on a $14: 10 \mathrm{~h}$ light:dark cycle and fed once daily with $\sim 3.5 \%$ body weight Gemma Micro 500 (Skretting USA). Embryos were obtained by natural spawning and were raised in embryo medium at $28.5^{\circ} \mathrm{C}$ and kept on a $14: 10 \mathrm{~h}$ light:dark cycle. All embryos used for experiments were obtained from pair-wise crosses and were staged according to (Kimmel et al., 1995). Exogenous food was provided starting at 5.5 days post fertilization (dpf) unless otherwise noted. Larvae were fed with GEMMA Micro 75 (Skretting) 3x a day until $14 \mathrm{dpf}$, GEMMA Micro 150 3x a day + Artemia 1x daily from $15 \mathrm{dpf}-42 \mathrm{dpf}$ and then GEMMA Micro 500 daily supplemented once a week with Artemia. Zebrafish sex is not determined until the juvenile stage, so gender is not a variable in experiments with embryos and larvae. Sex of adult fish included in analyses is noted in Figure legends. All zebrafish protocols were approved by the Carnegie Institution Department of Embryology Animal Care and Use Committee (Protocol \#139).

Stalactite (stl) mttp mutant zebrafish in the Tg(fli1:eGFP) ${ }^{y 1}$ background (Avraham-Davidi et al., 2012; Lawson and Weinstein, 2002; Yaniv et al., 2006) were provided by Karina Yaniv (Weizmann Institute of Science, Israel) and out-crossed to the AB wild-type strain. The stl mutation was maintained in both the presence and absence of the fli1:eGFP transgene. The c655 phenotype was identified in the Farber laboratory in the background of a kif7 mutant strain that was obtained from Philip Ingham (Lee Kong Chian School of Medicine, Singapore). The c655 mttp mutation was isolated from the kif7 mutation by out-crossing to the AB wild-type strain. The $c 655$ mutation was crossed into the $\operatorname{Tg}(f l i 1: e G F P)^{Y 1}$ reporter line. Both stl and $c 655$ mttp mutations were crossed into the ApoBb.1-NanoLuc LipoGlo reporter line (Thierer et al., In Press).

\section{Positional Cloning}

To map the location of the mutation responsible for the c655 phenotype, 23 embryos with normal yolks and 23 embryos with opaque yolks (3 dpf) were processed for RNA-seq (White et al., 2017). RNA was extracted from embryos by mechanical lysis in RLT buffer (Qiagen, 79216) containing $1 \mu \mathrm{L}$ of $14.3 \mathrm{M}$ beta-mercaptoethanol (Sigma, M6250). The lysate was combined with 1.8 volumes of Agencourt RNAClean XP (Beckman Coulter, A63987) beads and allowed to bind 
868 for 10 minutes. The plate was applied to a plate magnet (Invitrogen) until the solution cleared

869 and the supernatant was removed without disturbing the beads. This was followed by washing

870 the beads three times with $70 \%$ ethanol. After the last wash, the pellet was allowed to air dry for

87110 mins and then resuspended in $50 \mu$ of RNAse-free water. RNA was eluted from the beads

872 by applying the plate to the magnetic rack. RNA was quantified using the Quant-iT 610 RNA

873 assay (Invitrogen, Q33140). Total RNA from individual embryos was DNase treated for 20 mins

874 at $37^{\circ} \mathrm{C}$ followed by addition of $1 \mu \mathrm{L} 0.5 \mathrm{M}$ EDTA and inactivation at $75^{\circ} \mathrm{C}$ for 10 mins to remove

875 residual DNA. RNA was then cleaned using 2 volumes of Agencourt RNAClean XP (Beckman

876 Coulter, A63987) beads under the standard protocol. Strand-specific RNA-seq libraries

877 containing unique index sequences in the adapter were generated simultaneously following the

878 dUTP method using 700 ng total RNA and ERCC spike mix 2 (Ambion, 4456740). Libraries

879 were pooled and sequenced on Illumina HiSeq 2500 in 75bp paired-end mode. Sequence data

880 were deposited in European Nucleotide Archive under accession ERP023267. FASTQ files

881 were aligned to the GRCz10 reference genome using TopHat2 (Kim et al., 2013) (v2.0.13,

882 options: --library-type fr-firststrand). Ensembl 88 gene models were supplied to TopHat2 to aid

883 transcriptome mapping. MMAPPR (Hill et al., 2013) was used to determine the location of the

884 causal mutation. Variants were called from the pooled data using the GATK HaplotypeCaller

885 (Van der Auwera et al., 2013). Variants inside the regions output by MMAPPR were selected

886 and filtered for ones where the mutant sample was called as being homozygous alternate and

887 the siblings were heterozygous. The consequences of these variants on annotated genes was

888 calculated using the Ensembl Variant Effect Predictor (McLaren et al., 2016) and SIFT (Sim et

889 al., 2012). Variants with the following consequences were selected as candidates for the causal

890 mutation: stop_gained, splice_donor_variant, splice_acceptor_variant, transcript_ablation,

891 frameshift_variant, stop_lost, initiator_codon_variant, missense_variant, inframe_insertion,

892 inframe_deletion, transcript_amplification, splice_region_variant,

893 incomplete_terminal_codon_variant.

\section{DNA Extraction and Genotyping}

896 Genomic DNA was extracted from embryos or adult fin clips using a modified version of the

897 HotSHOT DNA extraction protocol (Meeker et al., 2007). Embryos/tissues were heated to $95^{\circ} \mathrm{C}$

898 for 18 minutes in $100 \mu \mathrm{L}$ of $50 \mathrm{mM} \mathrm{NaOH}$. The solution was cooled to $25^{\circ} \mathrm{C}$ and neutralized

899 with $10 \mu \mathrm{L}$ of $1 \mathrm{M}$ Tris- $\mathrm{HCl} \mathrm{pH} \mathrm{8.0.} \mathrm{Genotyping} \mathrm{primers} \mathrm{for} \mathrm{the} \mathrm{stalactite} \mathrm{allele} \mathrm{were} \mathrm{designed}$ 
using the dCAPS Finder 2.0 program (Neff et al., 2002) and synthesized by Eurofins Genomics.

901 The stalactite locus was amplified using the forward primer 5'-GTC TGA GGT TCA GAT GTA 902 CCT GTT AGG AC-3' and reverse primer 5'-CTC TGC TGT GAT GAG CGC AGG-3' (0.5 $\mu \mathrm{M}$

903 primer, $\mathrm{T}_{\mathrm{a}}=60^{\circ} \mathrm{C}$, extension time $\left.30^{\prime}\right)$. The forward primer introduces an Avall restriction site

904 into the mutant amplicon, such that following digestion (5 units of Avall (New England BioLabs,

$905 \mathrm{R} 0153$ ) at $37^{\circ} \mathrm{C}, 4 \mathrm{~h}$ ) the WT band is $157 \mathrm{bp}$, homozygous mutants have bands at $129 \mathrm{bp}$ and 28

$906 \mathrm{bp}$, and heterozygotes have all three bands. The c655 locus was amplified using the forward

907 primer 5'-AGAGACGGTGTCCAAGCAGG-3' and reverse primer 5'-GCTCAAAGACTTTCTTGC-

908 3' (0.25 $\mu \mathrm{M}$ primer, $\mathrm{Ta}=50^{\circ} \mathrm{C}$, extension time 30"). The $c 655$ mutation introduces a Bsrl

909 restriction site into the amplicon, such that following digestion (3 units of Bsrl (New England

910 BioLabs, R0527) in NEB Buffer 3.1 (B7203), $65^{\circ} \mathrm{C}, 3.5 \mathrm{~h}$ ) the WT band is $137 \mathrm{bp}$, homozygous

911 mutants have bands at $76 \mathrm{bp}$ and $61 \mathrm{bp}$, and heterozygotes have all three bands. For the

912 ApoBb.1-NanoLuc genotyping protocol, see (Thierer et al., In Press).

913

914 Generation of mttp-FLAG and ApoB48 plasmids

915 The wild-type zebrafish mttp coding sequence with a FLAG-tag prior to the termination codon at

916 the C-terminus was generated by custom gene synthesis and cloned into the pcDNA3.1+ vector

917 (mttp-FLAG)(Gene Universal Inc., Newark, DE). The stl and c655 mutations were subsequently

918 introduced to this plasmid by site-directed mutagenesis (Gene Universal Inc.) to generate

$919 m t t p^{s t}$-FLAG and $m t t p^{c 655}$-FLAG plasmids. The human pcDNA3.1-MTTP-FLAG plasmid was

920 synthesized as described previously (Rava et al., 2006; Sellers et al., 2003). The human

921 equivalent of the c655 mutation (G865V) was introduced into this plasmid using the Q5 Site-

922 directed mutagenesis kit (New England Biolabs), with the following primer pair: Forward 5'-

923 CGTATTAGCAgtaTGTGAATTCC-3', Reverse 5- CTTTCTTTTCTTTTCTGAGAG-3'. The

924 human ApoB48 sequence (Hussain et al., 1995) was cloned into the pcDNA3 under control of

925 the CMV promoter.

926

927

\section{Rescue of c655 opaque yolk phenotype}

$928 m t p^{c 655 / c 655}$ embryos were injected at the 1-cell stage with 20pg of zebrafish mttp-FLAG plasmid 929 and $20 \mathrm{pg}$ of CMV:eGFP-CAAX (synthesized using the Tol2kit Gateway cloning system using

930 the p5E-CMV/SP6, pME-eGFP-CAAX, and p3E-polyA entry clones (Kwan et al., 2007)) as a

931 marker of successful injections. Embryos were raised to $3 \mathrm{dpf}$ and screened for eGFP 
932 expression in the yolk sac. Images of eGFP+ control and experimental embryos were blinded

933 and scored for yolk opacity by another member of the lab.

\section{Ectopic sprout analysis}

$936 \mathrm{mttp}^{\text {st/stl }}, \mathrm{mttp}^{\mathrm{c655/c655}}$ and WT zebrafish in the $\mathrm{Tg}(\mathrm{fli1}: \mathrm{eGFP})^{y 1}$ background were imaged at $3 \mathrm{dpf}$

937 with a Zeiss Axiozoom V16 microscope equipped with a Zeiss PlanNeoFluar Z 1x/0.25 FWD

$93856 \mathrm{~mm}$ objective, AxioCam MRm camera, and Zen 2.5 software. The length of ectopic

939 angiogenic segments that extend from the subintestinal vessels were analyzed in Fiji

940 (Schindelin et al., 2012)(ImageJ V2.0.0, National Institutes of Health, USA) as described by

941 (Avraham-Davidi et al., 2012).

\section{Transmission Electron Microscopy}

944 Wild-type, $m t t p^{s t / s t l}, m t t p^{c 655 / c 655}$, and $m t t p^{\text {st/c655 }}$ mutant zebrafish embryos were fixed at $4 \mathrm{dpf}$ in a

$9453 \%$ glutaraldehyde, $1 \%$ formaldehyde, $0.1 \mathrm{M}$ cacodylate solution for $1-3 \mathrm{~h}$. Embryos were

946 trimmed and swim bladders were deflated before embedding in $2 \%$ low melt agarose and

947 processed as described in (Zeituni et al., 2016). Post-fixation was performed for $1 \mathrm{~h}$ with $1 \%$

948 osmium tetroxide $+1.25 \%$ potassium ferricyanide in cacodylate solution. Following $2 \times 10 \mathrm{~min}$

949 washes with water, samples were incubated with $0.05 \mathrm{M}$ maleate $\mathrm{pH} 6.5$ for $10 \mathrm{~min}$. Samples

950 were stained en bloc with $0.5 \%$ uranyl acetate in maleate for $4^{\circ} \mathrm{C}$ overnight. Following $2 \times 15$

951 min washes with water, samples were dehydrated through graded EtOH dilution (35\%, $2 \times 15$

$952 \mathrm{~min} ; 50 \%, 15 \mathrm{~min} ; 75 \%, 15 \mathrm{~min}$; 95\%, $15 \mathrm{~min} ; 100 \% 4 \times 15 \mathrm{~min}$ ). Samples were washed with

953 propylene oxide $4 \times 15$ min before incubation with 1:1 propylene oxide/resin (Epon 812 epoxy,

954 Ladd Research Industries, Williston, VT) for $1 \mathrm{~h}$ and evaporated overnight. This was followed

955 by $2 \times 1 \mathrm{~h}$ washes in $100 \%$ resin and a final embedding in $100 \%$ resin at $55^{\circ} \mathrm{C}$ overnight

956 followed by $70^{\circ} \mathrm{C}$ for three days. Sections were made on a Reichert Ultracut-S (Leica

957 Microsystems), mounted on naked 200 thin mesh grids, and stained with lead citrate. Images

958 were obtained with a Phillips Technai-12 electron microscope (FEI, Hillsboro, OR) and 794

959 Gatan multiscan CCD camera (Gatan, Pleasanton, CA) using Digital Micrograph software. Lipid

960 droplet number and area was quantified with Fiji. 


\section{Growth Time-Course}

965 Unsorted embryos from pair-wise in-crosses of stalactite or c655 heterozygous fish and pair-

966 wise crosses of $m t t p^{s t /+} \times m_{t t p}^{c 655 /+}$ were raised and were analyzed for standard length at 1, 3, 6,

967 9, 12, and 24 weeks post fertilization. At 1 week, fish were imaged using a Nikon SMZ1500

968 microscope with HR Plan Apo 1x WD 54 objective, Infinity 3 Lumenera camera and Infinity

969 Analyze 6.5 software. Standard length (Parichy et al., 2009) was measured using Fiji (NIH).

970 Starting at three weeks, standard length was measured with a ruler. Mass of the fish was also

971 measured starting at 6 weeks. At 1 and 3 weeks, gDNA was obtained from whole fish for

972 genotyping. At later time-points, genotyping was performed on fin clips. Images of fish at 12

973 weeks post fertilization were taken with a Canon T6 camera with a Canon EF 100mm Macro

974 Lens.

975

\section{Tissue Histology}

977 Adult zebrafish (7.5 mo; 2 males, 1 female per genotype) were placed individually into mating

978 tanks and fasted overnight $(\sim 24 \mathrm{~h})$. Fish were euthanized by submersion in ice-water. A piece

979 of the anterior intestine and the liver were dissected from each animal and fixed in neutral-

980 buffered formalin (Sigma, F8775) at $4^{\circ} \mathrm{C}$ for $48 \mathrm{~h}$. Sectioning and hematoxylin \& eosin staining

981 was performed by the Johns Hopkins University Oncology Tissue Services. Slides were imaged

982 with a Nikon E800 microscope with 60x/1.4 oil Plan Apo Nikon objective and Canon EOS T3

983 camera using EOS Utility image acquisition software.

\section{Tissue Lipid Extractions, HPLC \& Analysis}

986 Adult zebrafish (1 yr; 2-3 males, 2-3 females per genotype) were fasted overnight ( 24 h) and

987 euthanized by submersion in ice-water. Similar size pieces of the anterior intestine and the liver 988 were dissected from each animal and frozen on dry ice. Tissues were sonicated in $550 \mu \mathrm{L}$ of 989 homogenization buffer (20 mM Tris-HCl, $1 \mathrm{mM}$ EDTA), and the protein concentration of each 990 sample was measured using the BCA protein assay kit (Pierce, 23225). Lipids were extracted

991 from the remaining sample volume by a modified Bligh-Dyer procedure (Carten et al., 2011),

992 dried under vacuum, and re-suspended in $50 \mu \mathrm{L}$ of HPLC-grade isopropanol as the HPLC

993 injection solvent. Injection volumes were optimized for each sample to produce peak shapes

994 appropriate for quantitation (1-25 $\mu \mathrm{L})$. The lipid components of each sample were separated 995 and detected by an HPLC-CAD system using a LPG-3400RS quaternary pump, WPS-3000TRS 
autosampler (maintained at $20^{\circ} \mathrm{C}$ ), TCC-3000RS column oven (maintained at $40^{\circ} \mathrm{C}$ ), Accucore

997 C18 column (150 x $3.0 \mathrm{~mm}, 2.6 \mu \mathrm{m}$ particle size), FLD-3100 fluorescence detector (8 $\mathrm{mL}$ flow

998 cell maintained at $45^{\circ} \mathrm{C}$ ), and a Dionex Corona Veo charged aerosol detector (Thermo Fisher

999 Scientific). Lipids were separated over an $80 \mathrm{~min}$ time range in a multi-step mobile phase

1000 gradient as described in (Quinlivan et al., 2017). The lipid class and area of each analyte peak

1001 were determined using Chromeleon 7.2 (Thermo-Fisher Scientific) for chromatogram

1002 visualization and manual integration as described in (Otis et al., 2017). For quantitative

1003 comparisons between samples, each lipid peak area was normalized to the protein

1004 concentration of the homogenized tissue.

1005

1006 LipoGlo Assays

1007 All LipoGlo assays were performed with fish carrying a single copy of the LipoGlo

1008 (apoBb. $\left.{ }^{N L u c /+}\right)$ reporter. For detailed LipoGlo methods see (Thierer et al., In Press); Nano-Glo

1009 reporter system reagents are all from Promega Corp., (N1110; (Hall et al., 2012)). For

1010 quantitative assays and B-Ip size analysis, individual embryos were dispensed into 96-well

1011 plates (USAScientific, \#1402-9589) and homogenized in $100 \mu \mathrm{L}$ of B-Ip stabilization buffer (40

1012 mM EGTA, pH 8.0, 20\% sucrose + cOmplete mini, EDTA-free protease inhibitor (Sigma,

1013 11836170001)) by sonication in a microplate-horn sonicator (Qsonica Q700 sonicator with a

1014 Misonix CL-334 microplate horn assembly). Homogenate was stored on ice for immediate use

1015 or frozen at $-20^{\circ} \mathrm{C}$ for later use. ApoB-Nanoluc levels were quantified by mixing $40 \mu \mathrm{L}$ of

1016 embryo homogenate with an equal volume of diluted Nanoluc buffer (1:3 NanoGlo buffer:PBS +

$1017 \quad 0.5 \%$ NanoLuc substrate (furimazine)) in a 96-well opaque white OptiPlate (Perkin-Elmer,

1018 6005290), and the plate was read within 2 minutes of buffer addition using a SpectraMax M5

1019 plate reader (Molecular Devices) set to top-read chemiluminescent detection with a $500 \mathrm{~ms}$

1020 integration time. To quantify the size distribution of B-Ips, $12 \mu \mathrm{L}$ of homogenate was combined

1021 with $3 \mathrm{~mL}$ of $5 x$ loading dye (40\% sucrose, $0.25 \%$ bromophenol blue, in Tris/Borate/EDTA (TBE)

1022 buffer), and $12.5 \mathrm{~mL}$ of the resulting solution (10\% larval homogenate) was loaded per well on a

$10233 \%$ native polyacrylamide gel. Each gel included a migration standard of Di-I-labeled human

1024 LDL (L3482, ThermoFisher Scientific). Gels were run at 50V for $30 \mathrm{~min}$, followed by $125 \mathrm{~V}$ for 2

1025 h. Following application of $1 \mathrm{~mL}$ of TBE supplemented with $2 \mu \mathrm{L}$ of Nano-Glo substrate to the

1026 surface of the gel and incubating for $5 \mathrm{~min}$, gels were imaged with an Odyssey Fc (LI-COR

1027 Biosciences) gel imaging system. Images were obtained in the chemiluminescent channel (2 
1028 min exposure) and then the $600 \mathrm{~nm}$ channel $(30 \mathrm{sec}$ ) for Nanoluc detection and Di-I LDL

1029 standard detection, respectively. Each lane on the gel was converted to a plot profile in Fiji and

1030 divided into LDL, IDL, VLDL and Zero Mobility bins based on migration relative to the Di-I LDL

1031 standard. Pixel intensity from the plot profile was summed within each bin for comparison

1032 between genotypes. To determine the localization of B-Ips in the whole fish, intact embryos or

1033 larvae were anesthetized and fixed in 4\% paraformaldehyde for $3 \mathrm{~h}$ at room temperature.

1034 Following rinses in PBS + 0.1\% tween-20 (3 × 15 min), embryos were mounted in 1\% low-melt

1035 agarose (BP160-100, Fisher Scientific) in TBE supplemented with 1\% Nano-Glo substrate.

1036 Chemiluminescent images (10 and $30 \mathrm{sec}$ exposures with no illumination) and a brightfield

1037 image were taken with a Zeiss Axiozoom V16 microscope equipped with a Zeiss Plan NeoFluar

1038 Z 1x/0.25 FWD 56mm objective, AxioCam MRm camera, and Zen 2.5 software, using 2x2

1039 binning and 2x gain. Images were quantified using Fiji; regions of interest (ROI) were drawn on

1040 the brightfield image (viscera, trunk, and head), and these ROls were used to quantify the

1041 NanoLuc intensity on the 30sec exposure chemiluminescent images. ROls of the same shape

1042 were used to calculate the background signal, which was subtracted from the intensity value for 1043 each ROI.

1044

\section{Oil Red O staining}

1046 15-dpf larval zebrafish were fixed with 4\% paraformaldehyde in PBS for $3 \mathrm{~h}$ at room

1047 temperature and then overnight at $4^{\circ} \mathrm{C}$. Fish were rinsed in $60 \% 2$-propanol for 10 minutes, 1048 rocking and then put into 0.3\% Oil Red O (Sigma-Aldrich, \#O0625) to rock overnight at room

1049 temperature. Fish were rinsed 3 times with 60\% 2-propanol for 15 minutes. Washed fish were

1050 equilibrated step-wise into glycerol and imaged with incident light using a Nikon SMZ1500

1051 microscope with HR Plan Apo 1x WD 54 objective, Infinity 3 Lumenera camera, and Infinity

1052 Analyze 6.5 software.

1053

1054 ApoB secretion assays

1055 Monkey kidney COS-7 cells which do not express MTTP or ApoB were plated in $10 \mathrm{~cm}^{2}$ cell

1056 culture dishes at a density of $9 \times 10^{5}$ cells per plate and grown in Dulbecco's modified Eagle's

1057 medium (DMEM) containing $10 \%$ fetal bovine serum, L-glutamine, and antibiotics at $37^{\circ} \mathrm{C}$.

1058 COS-7 cells were transfected with $5 \mu \mathrm{g}$ of plasmid expressing human ApoB48 cDNA under the

1059 control of CMV promoter using endofectin (Genecopoeia, EF014) according to the 
1060 manufacturer's protocol. After 24 hours, cells from each dish were harvested, equally distributed

1061 in 6-well plates, and reverse transfected with $3 \mu \mathrm{g}$ of either pcDNA3, pcDNA3-mttp-FLAG,

1062 pcDNA3-mttp ${ }^{s t l}$-FLAG, pcDNA3-mttp ${ }^{c 655}$-FLAG, pcDNA3-MTTP-FLAG, or pcDNA3-

1063 MTTP(G865V)-FLAG plasmids. After $32 \mathrm{~h}$ cells were incubated overnight with $1 \mathrm{~mL}$ of DMEM

1064 containing 10\%FBS. The overnight conditioned media were collected to measure ApoB by

1065 ELISA (Bakillah et al., 1997; Hussain et al., 1995). Cells were scraped in PBS and a small

1066 aliquot was used to measure total protein using a Coomassie protein assay (Thermo Scientific,

1067 \#1856209). Cells were lysed in cell extract buffer (100 mM Tris, pH 7.4, $150 \mathrm{mM} \mathrm{NaCl,} 1 \mathrm{mM}$

1068 EGTA, 1mM EDTA, 1\% Triton X-100, 0.5\% sodium deoxycholate). Lysates were rotated for $1 \mathrm{~h}$

1069 at $4{ }^{\circ} \mathrm{C}$ to solubilize the membranes and centrifuged at $16,000 \mathrm{~g}$ for 30 mins. ApoB was

1070 measured in the supernatant via ELISA. Briefly, high binding 96 well plates (Corning, \#3366)

1071 were incubated with capture antibody anti-LDL (apoB), clone 1D1(MyBiosource, \#MBS465020,

1072 1:1000 dilution) overnight at room temperature. The plate was washed $3 x$ with PBS-T (PBS +

1073 0.05\% Tween-20) and blocked with 3\% BSA (Boston Bio Products, \#P753) for $1 \mathrm{~h}$ and washed

$10743 x$ with PBS-T, before incubating with $100 \mu \mathrm{L}$ of standards and experimental samples for $3 \mathrm{~h}$.

1075 The plate was washed 3x with PBST and incubated with $100 \mu \mathrm{L}$ of human ApoB antibody

1076 (Academy Bio-Medical Company, Inc., \#20S-G2, 1:1000 dilution) for $1 \mathrm{~h}$. After washing the

1077 plate $3 \mathrm{x}$ with PBS-T, $100 \mu \mathrm{L}$ of alkaline phosphatase labeled anti-goat IgG (Southern Biotech,

1078 \#6300-04, 1:3000 dilution) was added to each well and incubated for $1 \mathrm{~h}$. The plate was

1079 washed 3x with Diethanolamine buffer, pH 9.5 and $100 \mu$ of PNPP (Thermo Scientific, \#34045,

$10801 \mathrm{mg} / \mathrm{mL}$ ) was added to each well before reading the plate at $405 \mathrm{~nm}$ in a PerkinElmer Victor ${ }^{3}$

10811420 multilabel counter. Data for zebrafish and human plasmids were obtained in the same

1082 experiments, but are graphed separately in figures $5 A, B \& 6 B, C$; the pcDNA control data is

1083 displayed in both sets of graphs.

1084

1085 Immunofluorescence

1086 COS-7 cells were plated at a density of 50,000 cells on coverslips in 12-well dishes and

1087 transfected with $2 \mu \mathrm{g}$ of plasmids expressing either zebrafish or human MTTP-FLAG plasmids.

1088 After $48 \mathrm{~h}$, cells were fixed in paraformaldehyde and blocked with PBS supplemented with $1 \mathrm{mM}$

$1089 \mathrm{MgCl}_{2}, 0.5 \mathrm{mM} \mathrm{CaCl}_{2}, 3 \% \mathrm{BSA}, 0.1 \%$ Triton X-100 and 1\% horse serum. Cells were incubated

1090 with anti-FLAG M2 monoclonal antibody (Sigma \# F3165, 1:250 dilution) and anti-calnexin

1091 antibody (Santa Cruz Biotechnology, \# sc-11397, 1:250 dilution) overnight. Cells were washed 
1092 three times with PBS and incubated with goat anti-mouse Alexa Fluor-594 (Invitrogen, \#A11005, 1093 1:500 dilution) and donkey anti-rabbit Alexa-Fluor-488 (Invitrogen, \# A21206, 1:500 dilution) for

$10941 \mathrm{~h}$. The cells were washed and mounted with Vectashield mounting medium (Vector

1095 Laboratories, \#H-1000). Images were taken on a Leica SP5II confocal microscope with a

1096 63x1.4 HCX PL Apo oil immersion lens.

\section{Immunoprecipitation and Western blotting}

1099 Transfected COS-7 cells were washed three times with ice cold PBS and scraped in buffer K (1 $1100 \mathrm{mM}$ Tris- $\mathrm{HCl}, 1 \mathrm{mM}$ EGTA and $1 \mathrm{mM} \mathrm{MgCl}_{2}, \mathrm{pH}$ 7.6) containing protease inhibitor cocktail 1101 (Sigma, \# P2714). Cells were mechanically lysed by passing them 10 times with $30_{1 / 2}$-gauge 1102 needle and small fractions were used to measure the total protein using a Coomassie protein 1103 assay (Thermo Fisher Scientific, \#1856209). Cell lysate was incubated with Anti-FLAG M2 1104 antibody for $1 \mathrm{~h}$ and immunoprecipitated (IP) using (protein A/G) agarose beads (Santa-Cruz 1105 Biotechnology, \# SC2003). The supernatants were used to detect actin via Western blotting 1106 and served as loading controls. Both the supernatant and immunoprecipitated fractions were 1107 subjected to electrophoresis on an 8\% SDS-PAGE gel. The weight separated proteins were 1108 transferred to nitrocellulose membranes and probed with either anti-FLAG M2 (1:1000) or anti1109 actin (Thermo Fisher Scientific, \#PA1-183, (1:3000)) prepared in 2\% BSA in TBS. The blots 1110 were washed and probed with HRP-conjugated corresponding secondary antibodies (goat anti1111 rabbit, Cell Signaling Technology, \#7074, 1:5000 or goat anti-mouse, Thermo Fisher Scientific, 1112 \#62-6520, 1:5000). The blots were developed in ChemiDoc ${ }^{\mathrm{TM}}$-Touch Imaging system from Bio 1113 Rad.

\section{Triglyceride transfer assay}

1116 Following transfection with plasmids as described above, cell lysate (35 $\mu \mathrm{g})$ prepared in buffer $\mathrm{K}$ 1117 containing protease inhibitor cocktail was incubated with donor vesicles containing NBD-labeled 1118 triolein (Setareh Biotech, LLC, \#6285) and acceptor vesicles. Fluorescence was measured at 1119 different time intervals $(5,10,15,30,45$ and 60 mins). Percent triglyceride transfer was 1120 calculated after subtracting the blank and dividing it by the total fluorescence reading obtained 1121 by disrupting vesicles with isopropanol, as described previously (Athar et al., 2004; Rava et al., 1122 2005). Where noted, assays also included the MTTP inhibitor Lomitapide (Aegerion

1123 Pharmaceuticals, \#AEGR-733) at a concentration of $1 \mu \mathrm{M}$. 


\section{Phospholipid transfer assay}

1126 COS-7 cells were transfected with $9 \mu \mathrm{g}$ of either zebrafish mttp-FLAG or human MTTP-FLAG

1127 plasmids in $10 \mathrm{~cm}^{2}$ cell culture dishes. After $48 \mathrm{~h}$, cell lysates were prepared in buffer $\mathrm{K}$

1128 containing protease inhibitor cocktail (Sigma, \#P2714). The cell lysates were centrifuged at

$112912,000 \mathrm{~g}$ for $10 \mathrm{mins}$ at $4^{\circ} \mathrm{C}$. A small aliquot of cell lysate was used for measuring protein and

1130 kept for Western blotting to measure expression level. Equal concentrations of protein from

1131 each sample were incubated with $40 \mu \mathrm{L}$ of M2 agarose beads (Sigma, \#A2220) for $3 \mathrm{~h}$ at $4^{\circ} \mathrm{C}$.

1132 FLAG-tagged protein were eluted twice with $200 \mathrm{ng} / \mu \mathrm{L}$ FLAG peptide (Sigma, \#F3290; $1 \mathrm{~h}$ at

$\left.11334^{\circ} \mathrm{C}\right)$. PL transfer activity was assayed using NBD labeled Phosphoethanolamine,

1134 triethylammonium (Thermo Fisher Scientific, \#N360). The purified FLAG-tagged proteins were

1135 incubated with donor vesicles containing NBD-Phosphoethanolamine and acceptor vesicles.

1136 The fluorescence was measured at different time intervals (1, 2, 3 and $4 \mathrm{~h}$ ). The percentage

1137 transfer of phospholipid was calculated as the difference between the fluorescence reading at

1138 the $0 \mathrm{~h}$ time point and $3 \mathrm{~h}$ time point divided by the total fluorescence reading obtained by

1139 disrupting vesicles with isopropanol as described previously (Athar et al., 2004; Rava et al.,

1140 2005). Where noted, assays also included the MTTP inhibitor Lomitapide at a concentration of

$11411 \mu \mathrm{M}$.

1142

\section{$1143 \quad$ Modeling}

1144 Predicted models of human MTTP and zebrafish Mttp proteins were generated using Phyre2

1145 (Kelley et al., 2015) and the graphics were enhanced for clarity using Chimera (UCSF Chimera, 1146 developed by the Resource for Biocomputing, Visualization, and Informatics at the University of

1147 California, San Francisco, with support from NIH P41-GM103311 (Pettersen et al., 2004).

1149 Statistical Analyses

1150 Graphing and some statistics, including One-way, randomized block and Repeated Measures

1151 ANOVA with Bonferroni post-hoc tests, Kruskall-Wallis with Dunn's Multiple Comparison test

1152 and Chi-square tests were performed with GraphPad Prism (GraphPad Software). When

1153 sample sizes and variance between groups were significantly different, Robust ANOVA was

1154 performed using $\mathrm{R}$ to determine overall significance of noted datasets (Mair and Wilcox,

1155 2018)(https://cran.r-project.org/web/packages/WRS2/vignettes/WRS2.pdf),((Mangiafico, 2015),

1156 https://rcompanion.org/rcompanion/d_08a.html). When significant differences were present

1157 between genotypes, Games-Howell post-hoc tests were used to make pair-wise comparisons at 
1158 each time point using SPSS Statistics (IBM), adjusting the significance level for multiple

1159 comparisons. Details of the statistical analyses can be found either in the figure legend or

1160 results sections. Sample sizes for each experiment are indicated in the figure legends for each

1161 experiment.

1162

\section{Additional Software}

1164 DNA, mRNA, and protein sequence alignments were performed with MacVector V15.5

1165 (MacVector, Inc.). Microsoft Word and Excel were used for manuscript preparation and data 1166 analysis, respectively, figures were assembled in Adobe Illustrator CS5 (Adobe Systems) and

1167 references were assembled with EndNote 8X.

1168

1169

1170

1171

1172

1173

1174

1175

1176

1177

1178

1179

1180

1181

1182

1183

1184

1185

1186

1187

1188

1189

1190

1191

1192

1193

\section{REFERENCES}

Alexander, C.A., Hamilton, R.L., and Havel, R.J. (1976). Subcellular localization of B apoprotein of plasma lipoproteins in rat liver. J Cell Biol 69, 241-263.

Anderson, T.A., Levitt, D.G., and Banaszak, L.J. (1998). The structural basis of lipid interactions in lipovitellin, a soluble lipoprotein. Structure 6, 895-909.

Athar, H., Iqbal, J., Jiang, X.C., and Hussain, M.M. (2004). A simple, rapid, and sensitive fluorescence assay for microsomal triglyceride transfer protein. J Lipid Res 45, 764-772.

Atzel, A., and Wetterau, J.R. (1993). Mechanism of microsomal triglyceride transfer protein catalyzed lipid transport. Biochemistry 32, 10444-10450.

Atzel, A., and Wetterau, J.R. (1994). Identification of two classes of lipid molecule binding sites on the microsomal triglyceride transfer protein. Biochemistry 33, 15382-15388.

Austin, M.A., Breslow, J.L., Hennekens, C.H., Buring, J.E., Willett, W.C., and Krauss, R.M. (1988). Low-density lipoprotein subclass patterns and risk of myocardial infarction. JAMA 260, 1917-1921.

Avraham-Davidi, I., Ely, Y., Pham, V.N., Castranova, D., Grunspan, M., Malkinson, G., GibbsBar, L., Mayseless, O., Allmog, G., Lo, B., et al. (2012). ApoB-containing lipoproteins regulate angiogenesis by modulating expression of VEGF receptor 1. Nat Med 18, 967-973.

Bakillah, A., Zhou, Z., Luchoomun, J., and Hussain, M.M. (1997). Measurement of apolipoprotein $B$ in various cell lines: correlation between intracellular levels and rates of secretion. Lipids 32, 1113-1118.

Banaszak, L., Sharrock, W., and Timmins, P. (1991). Structure and function of a lipoprotein: lipovitellin. Annu Rev Biophys Biophys Chem 20, 221-246. 
Berthier, M.T., Couture, P., Houde, A., Paradis, A.M., Sammak, A., Verner, A., Depres, J.P., Gagne, C., Gaudet, D., and Vohl, M.C. (2004). The c.419-420insA in the MTP gene is associated with abetalipoproteinemia among French-Canadians. Mol Genet Metab 81, 140-143.

1197 Bjornheden, T., Babyi, A., Bondjers, G., and Wiklund, O. (1996). Accumulation of lipoprotein 1198 fractions and subfractions in the arterial wall, determined in an in vitro perfusion system.

1199 Atherosclerosis 123, 43-56.

Blom, D.J., Averna, M.R., Meagher, E.A., du Toit Theron, H., Sirtori, C.R., Hegele, R.A., Shah, P.K., Gaudet, D., Stefanutti, C., Vigna, G.B., et al. (2017). Long-Term Efficacy and Safety of the Microsomal Triglyceride Transfer Protein Inhibitor Lomitapide in Patients With Homozygous Familial Hypercholesterolemia. Circulation 136, 332-335.

Boren, J., Rustaeus, S., and Olofsson, S.O. (1994). Studies on the assembly of apolipoprotein B-100- and B-48-containing very low density lipoproteins in McA-RH7777 cells. J Biol Chem 269, 25879-25888.

1207 Bradbury, P., Mann, C.J., Kochl, S., Anderson, T.A., Chester, S.A., Hancock, J.M., Ritchie, P.J., 1208 Amey, J., Harrison, G.B., Levitt, D.G., et al. (1999). A common binding site on the microsomal 1209 triglyceride transfer protein for apolipoprotein $B$ and protein disulfide isomerase. J Biol Chem $1210 \quad 274,3159-3164$.

1211 Carten, J.D., Bradford, M.K., and Farber, S.A. (2011). Visualizing digestive organ morphology 1212 and function using differential fatty acid metabolism in live zebrafish. Dev Biol 360, 276-285.

1213 Carvalho, L., and Heisenberg, C.P. (2010). The yolk syncytial layer in early zebrafish 1214 development. Trends Cell Biol 20, 586-592.

1215 CDC (2018). Centers for Disease Control and Prevention, National Center for Health Statistics. Underlying Cause of Death 1999-2017 on CDC WONDER Online Database, released December, 2018. Data are from the Multiple Cause of Death Files, 1999-2017, as compiled from data provided by the 57 vital statistics jurisdictions through the Vital Statistics Cooperative Program. In http://wonder.cdc.gov/ucd-icd10.html (Centers for Disease Control).

1220 Cuchel, M., Bloedon, L.T., Szapary, P.O., Kolansky, D.M., Wolfe, M.L., Sarkis, A., Millar, J.S., 1221 Ikewaki, K., Siegelman, E.S., Gregg, R.E., et al. (2007). Inhibition of microsomal triglyceride 1222 transfer protein in familial hypercholesterolemia. N Engl J Med 356, 148-156.

1223 Cuchel, M., Bruckert, E., Ginsberg, H.N., Raal, F.J., Santos, R.D., Hegele, R.A., Kuivenhoven, 1224 J.A., Nordestgaard, B.G., Descamps, O.S., Steinhagen-Thiessen, E., et al. (2014). Homozygous familial hypercholesterolaemia: new insights and guidance for clinicians to improve detection and clinical management. A position paper from the Consensus Panel on Familial

1228 Cuchel, M., Meagher, E.A., du Toit Theron, H., Blom, D.J., Marais, A.D., Hegele, R.A., Averna, 1229 M.R., Sirtori, C.R., Shah, P.K., Gaudet, D., et al. (2013). Efficacy and safety of a microsomal 1230 triglyceride transfer protein inhibitor in patients with homozygous familial hypercholesterolaemia: 1231 a single-arm, open-label, phase 3 study. Lancet 381, 40-46. 
1232 Davidson, N.O., and Shelness, G.S. (2000). APOLIPOPROTEIN B: mRNA editing, lipoprotein assembly, and presecretory degradation. Annu Rev Nutr 20, 169-193.

1234 Di Filippo, M., Collardeau Frachon, S., Janin, A., Rajan, S., Marmontel, O., Decourt, C., Rubio, 1235 A., Nony, S., Dumont, S., Cuerq, C., et al. (2019). Normal serum ApoB48 and red cells vitamin 1236 E concentrations after supplementation in a novel compound heterozygous case of

1237 abetalipoproteinemia. Atherosclerosis 284, 75-82.

1238

1239

1240

1241

1242

1243

1244

1245

1246

1247

1248

1249

1250

1251

1252

1253

1254

1255

1256

1257

1258

1259

1260

1261

1262

1263

1264

1265

1266

1267

1268

Di Filippo, M., Crehalet, H., Samson-Bouma, M.E., Bonnet, V., Aggerbeck, L.P., Rabes, J.P., Gottrand, F., Luc, G., Bozon, D., and Sassolas, A. (2012). Molecular and functional analysis of two new MTTP gene mutations in an atypical case of abetalipoproteinemia. J Lipid Res 53, 548555.

Di Leo, E., Lancellotti, S., Penacchioni, J.Y., Cefalu, A.B., Averna, M., Pisciotta, L., Bertolini, S., Calandra, S., Gabelli, C., and Tarugi, P. (2005). Mutations in MTP gene in abeta- and hypobetalipoproteinemia. Atherosclerosis 180, 311-318.

Dougan, S.K., Salas, A., Rava, P., Agyemang, A., Kaser, A., Morrison, J., Khurana, A., Kronenberg, M., Johnson, C., Exley, M., et al. (2005). Microsomal triglyceride transfer protein lipidation and control of CD1d on antigen-presenting cells. J Exp Med 202, 529-539.

Elovson, J., Chatterton, J.E., Bell, G.T., Schumaker, V.N., Reuben, M.A., Puppione, D.L., Reeve, J.R., Jr., and Young, N.L. (1988). Plasma very low density lipoproteins contain a single molecule of apolipoprotein B. J Lipid Res 29, 1461-1473.

Farese, R.V., Jr., Cases, S., Ruland, S.L., Kayden, H.J., Wong, J.S., Young, S.G., and Hamilton, R.L. (1996). A novel function for apolipoprotein B: lipoprotein synthesis in the yolk sac is critical for maternal-fetal lipid transport in mice. J Lipid Res 37, 347-360.

FDA (2012). JUXTAPID TM (lomitapide) capsules, for oral use Initial U.S. Approval: 2012 In https://www.accessdata.fda.gov/drugsatfda docs/label/2012/203858s000lb1.Pdf.

Fraher, D., Sanigorski, A., Mellett, N.A., Meikle, P.J., Sinclair, A.J., and Gibert, Y. (2016). Zebrafish Embryonic Lipidomic Analysis Reveals that the Yolk Cell Is Metabolically Active in Processing Lipid. Cell Rep 14, 1317-1329.

Glickman, R.M., Khorana, J., and Kilgore, A. (1976). Localization of apolipoprotein B in intestinal epithelial cells. Science 193, 1254-1255.

Hall, M.P., Unch, J., Binkowski, B.F., Valley, M.P., Butler, B.L., Wood, M.G., Otto, P., Zimmerman, K., Vidugiris, G., Machleidt, T., et al. (2012). Engineered luciferase reporter from a deep sea shrimp utilizing a novel imidazopyrazinone substrate. ACS Chem Biol 7, 1848-1857.

Hamilton, R.L., Wong, J.S., Cham, C.M., Nielsen, L.B., and Young, S.G. (1998). Chylomicronsized lipid particles are formed in the setting of apolipoprotein B deficiency. J Lipid Res 39, 1543-1557.

Hill, J.T., Demarest, B.L., Bisgrove, B.W., Gorsi, B., Su, Y.C., and Yost, H.J. (2013). MMAPPR: mutation mapping analysis pipeline for pooled RNA-seq. Genome Res 23, 687-697. 
Hiramatsu, N., Todo, T., Sullivan, C.V., Schilling, J., Reading, B.J., Matsubara, T., Ryu, Y.W., Mizuta, H., Luo, W., Nishimiya, O., et al. (2015). Ovarian yolk formation in fishes: Molecular mechanisms underlying formation of lipid droplets and vitellogenin-derived yolk proteins. Gen Comp Endocrinol 221, 9-15.

1273 Hussain, M.M., and Bakillah, A. (2008). New approaches to target microsomal triglyceride 1274 transfer protein. Curr. Opin. Lipidol 19, 572-578.

1275 Hussain, M.M., Iqbal, J., Anwar, K., Rava, P., and Dai, K. (2003a). Microsomal triglyceride 1276 transfer protein: a multifunctional protein. Front Biosci 8, s500-506.

1277 Hussain, M.M., Kancha, R.K., Zhou, Z., Luchoomun, J., Zu, H., and Bakillah, A. (1996). Chylomicron assembly and catabolism: role of apolipoproteins and receptors. Biochim Biophys 1279 Acta 1300, 151-170.

1280 Hussain, M.M., Shi, J., and Dreizen, P. (2003b). Microsomal triglyceride transfer protein and its role in apoB-lipoprotein assembly. J Lipid Res 44, 22-32.

1282 Hussain, M.M., Zhao, Y., Kancha, R.K., Blackhart, B.D., and Yao, Z. (1995). Characterization of recombinant human apoB-48-containing lipoproteins in rat hepatoma McA-RH7777 cells transfected with apoB-48 cDNA. Overexpression of apoB-48 decreases synthesis of endogenous apoB-100. Arterioscler Thromb Vasc Biol 15, 485-494. J.V. (2018). Light transmission/absorption characteristics of the meibomian gland. Ocul Surf 16, 1288 448-453. lqbal, J., Walsh, M.T., Hammad, S.M., Cuchel, M., Tarugi, P., Hegele, R.A., Davidson, N.O., Rader, D.J., Klein, R.L., and Hussain, M.M. (2015). Microsomal Triglyceride Transfer Protein Transfers and Determines Plasma Concentrations of Ceramide and Sphingomyelin but Not Glycosylceramide. J Biol Chem 290, 25863-25875.

1293 Ivanova, E.A., Myasoedova, V.A., Melnichenko, A.A., Grechko, A.V., and Orekhov, A.N. (2017). 1294 Small Dense Low-Density Lipoprotein as Biomarker for Atherosclerotic Diseases. Oxid Med Cell 1295 Longev 2017, 1273042.

1296 Jamil, H., Dickson, J.K., Jr., Chu, C.H., Lago, M.W., Rinehart, J.K., Biller, S.A., Gregg, R.E., and Wetterau, J.R. (1995). Microsomal triglyceride transfer protein. Specificity of lipid binding and 1298 transport. J Biol Chem 270, 6549-6554.

1299 Jamil, H., Gordon, D.A., Eustice, D.C., Brooks, C.M., Dickson, J.K., Jr., Chen, Y., Ricci, B., Chu, 1300 C.H., Harrity, T.W., Ciosek, C.P., Jr., et al. (1996). An inhibitor of the microsomal triglyceride 1301 transfer protein inhibits apoB secretion from HepG2 cells. Proc Natl Acad Sci U S A 93, 11991130211995.

1303 Kane, J.P., Hardman, D.A., and Paulus, H.E. (1980). Heterogeneity of apolipoprotein B: 1304 isolation of a new species from human chylomicrons. Proc Natl Acad Sci U S A 77, 2465-2469. 
1305

1306

1307

1308

1309

1310

1311

1312

1313

1314

1315

1316

1317

1318

1319

1320

1321

1322

1323

1324

1325

1326

1327

1328

1329

1330

1331

1332

1333

1334

1335

1336

1337

1338

1339

1340

1341

Kane, J.P.H., R. J. (1995). Disorders of the biogenesis and secretion of lipoproteins containing the B apolipoproteins. In The metabolic and molecular bases of inherited disorders. A.L.B. C. R. Scriver, W. S. Sly and D. Valle., ed. (New York: McGraw-Hill, Inc.), pp. 1853-1885.

Kelley, L.A., Mezulis, S., Yates, C.M., Wass, M.N., and Sternberg, M.J. (2015). The Phyre2 web portal for protein modeling, prediction and analysis. Nat Protoc 10, 845-858.

Kessler, J.I., Stein, J., Dannacker, D., and Narcessian, P. (1970). Biosynthesis of low density lipoprotein by cell-free preparations of rat intestinal mucosa. J Biol Chem 245, 5281-5288.

Khatun, I., Walsh, M.T., and Hussain, M.M. (2013). Loss of both phospholipid and triglyceride transfer activities of microsomal triglyceride transfer protein in abetalipoproteinemia. J Lipid Res $54,1541-1549$.

Khatun, I., Zeissig, S., Iqbal, J., Wang, M., Curiel, D., Shelness, G.S., Blumberg, R.S., and Hussain, M.M. (2012). Phospholipid transfer activity of microsomal triglyceride transfer protein produces apolipoprotein $B$ and reduces hepatosteatosis while maintaining low plasma lipids in mice. Hepatology 55, 1356-1368.

Kim, D., Pertea, G., Trapnell, C., Pimentel, H., Kelley, R., and Salzberg, S.L. (2013). TopHat2: accurate alignment of transcriptomes in the presence of insertions, deletions and gene fusions. Genome Biol 14, R36.

Kimmel, C.B., Ballard, W.W., Kimmel, S.R., Ullmann, B., and Schilling, T.F. (1995). Stages of embryonic development of the zebrafish. Dev Dyn 203, 253-310.

Kimmel, C.B., and Law, R.D. (1985). Cell lineage of zebrafish blastomeres. II. Formation of the yolk syncytial layer. Dev Biol 108, 86-93.

Kulinski, A., Rustaeus, S., and Vance, J.E. (2002). Microsomal triacylglycerol transfer protein is required for lumenal accretion of triacylglycerol not associated with $A p o B$, as well as for $A p o B$ lipidation. J Biol Chem 277, 31516-31525.

Kwan, K.M., Fujimoto, E., Grabher, C., Mangum, B.D., Hardy, M.E., Campbell, D.S., Parant, J.M., Yost, H.J., Kanki, J.P., and Chien, C.B. (2007). The Tol2kit: a multisite gateway-based construction kit for Tol2 transposon transgenesis constructs. Dev Dyn 236, 3088-3099.

Lawson, N.D., and Weinstein, B.M. (2002). In vivo imaging of embryonic vascular development using transgenic zebrafish. Dev Biol 248, 307-318.

Lee, J., and Hegele, R.A. (2014). Abetalipoproteinemia and homozygous hypobetalipoproteinemia: a framework for diagnosis and management. J Inherit Metab Dis 37, 333-339.

Madsen, E.M., Lindegaard, M.L., Andersen, C.B., Damm, P., and Nielsen, L.B. (2004). Human placenta secretes apolipoprotein B-100-containing lipoproteins. J Biol Chem 279, 55271-55276.

Mahley, R.W., Bersot, T.P., LeQuire, V.S., Levy, R.I., Windmueller, H.G., and Brown, W.V. (1970). Identity of very low density lipoprotein apoproteins of plasma and liver Golgi apparatus. Science 168, 380-382. 
1342 Mair, P., and Wilcox, R. (2018). Robust Statistical Methods Using WRS2. In https://cran.r1343 project.org/web/packages/WRS2/vignettes/WRS2.pdf.

1344 Mangiafico, S.S. (2015). An R Companion for the Handbook of Biological Statistics, version 1345 1.3.2. In https://rcompanion.org/rcompanion/d 08a.html.

1346

1347

1348

1349

1350

1351

1352

1353

1354

1355

1356

1357

1358

1359

1360

1361

1362

1363

1364

1365

1366

1367

1368

1369

1370

1371

1372

1373

1374

1375

1376

1377

Mani-Ponset, L., Guyot, E., Diaz, J., and Connes, R. (1996). Utilization of yolk reserves during post-embryonic development in three teleostean species: the sea bream Sparus aurata, the sea bass Dicentrarchus labrax, and the pike-perch Stizostedion lucioperca. Marine Biology 126, 539-547.

Mann, C.J., Anderson, T.A., Read, J., Chester, S.A., Harrison, G.B., Kochl, S., Ritchie, P.J., Bradbury, P., Hussain, F.S., Amey, J., et al. (1999). The structure of vitellogenin provides a molecular model for the assembly and secretion of atherogenic lipoproteins. J Mol Biol 285, 391-408.

Marza, E., Barthe, C., Andre, M., Villeneuve, L., Helou, C., and Babin, P.J. (2005). Developmental expression and nutritional regulation of a zebrafish gene homologous to mammalian microsomal triglyceride transfer protein large subunit. Dev Dyn 232, 506-518.

McLaren, W., Gil, L., Hunt, S.E., Riat, H.S., Ritchie, G.R., Thormann, A., Flicek, P., and Cunningham, F. (2016). The Ensembl Variant Effect Predictor. Genome Biol 17, 122.

Meeker, N.D., Hutchinson, S.A., Ho, L., and Trede, N.S. (2007). Method for isolation of PCRready genomic DNA from zebrafish tissues. Biotechniques 43, 610, 612, 614.

Michels, R., Foschum, F., and Kienle, A. (2008). Optical properties of fat emulsions. Opt Express 16, 5907-5925.

Miller, S.A., Burnett, J.R., Leonis, M.A., McKnight, C.J., van Bockxmeer, F.M., and Hooper, A.J. (2014). Novel missense MTTP gene mutations causing abetalipoproteinemia. Biochim Biophys Acta 1842, 1548-1554.

Miyares, R.L., de Rezende, V.B., and Farber, S.A. (2014). Zebrafish yolk lipid processing: a tractable tool for the study of vertebrate lipid transport and metabolism. Dis Model Mech 7, 915927.

Narcisi, T.M., Shoulders, C.C., Chester, S.A., Read, J., Brett, D.J., Harrison, G.B., Grantham, T.T., Fox, M.F., Povey, S., de Bruin, T.W., et al. (1995). Mutations of the microsomal triglyceride-transfer-protein gene in abetalipoproteinemia. Am J Hum Genet 57, 1298-1310.

Neff, M.M., Turk, E., and Kalishman, M. (2002). Web-based primer design for single nucleotide polymorphism analysis. Trends Genet 18,613-615.

Olzmann, J.A., and Carvalho, P. (2019). Dynamics and functions of lipid droplets. Nat Rev Mol Cell Biol 20, 137-155.

Otis, J.P., and Farber, S.A. (2016). High-fat Feeding Paradigm for Larval Zebrafish: Feeding, Live Imaging, and Quantification of Food Intake. J Vis Exp. 
Otis, J.P., Shen, M.C., Quinlivan, V., Anderson, J.L., and Farber, S.A. (2017). Intestinal epithelial cell caveolin 1 regulates fatty acid and lipoprotein cholesterol plasma levels. Dis Model Mech 10, 283-295.

1381 Otis, J.P., Zeituni, E.M., Thierer, J.H., Anderson, J.L., Brown, A.C., Boehm, E.D., Cerchione, 1382 D.M., Ceasrine, A.M., Avraham-Davidi, I., Tempelhof, H., et al. (2015). Zebrafish as a model for 1383 apolipoprotein biology: comprehensive expression analysis and a role for ApoA-IV in regulating 1384 food intake. Dis Model Mech 8, 295-309. Parichy, D.M., Elizondo, M.R., Mills, M.G., Gordon, T.N., and Engeszer, R.E. (2009). Normal table of postembryonic zebrafish development: staging by externally visible anatomy of the living fish. Dev Dyn 238, 2975-3015. protein and apolipoprotein $B$ within the endoplasmic reticulum in a heterologous expression system. J Biol Chem 271, 18686-18694.

1391 Pettersen, E.F., Goddard, T.D., Huang, C.C., Couch, G.S., Greenblatt, D.M., Meng, E.C., and 1392 Ferrin, T.E. (2004). UCSF Chimera--a visualization system for exploratory research and 1393 analysis. J Comput Chem 25, 1605-1612.

1394 Plonne, D., Winkler, L., Franke, H., and Dargel, R. (1992). The visceral yolk sac--an important site of synthesis and secretion of apolipoprotein B containing lipoproteins in the feto-placental unit of the rat. Biochim Biophys Acta 1127, 174-185.

Quinlivan, V.H., Wilson, M.H., Ruzicka, J., and Farber, S.A. (2017). An HPLC-CAD/fluorescence lipidomics platform using fluorescent fatty acids as metabolic tracers. J Lipid Res 58, 10081020.

1400 Raabe, M., Flynn, L.M., Zlot, C.H., Wong, J.S., Veniant, M.M., Hamilton, R.L., and Young, S.G. (1998). Knockout of the abetalipoproteinemia gene in mice: reduced lipoprotein secretion in heterozygotes and embryonic lethality in homozygotes. Proc Natl Acad Sci U S A 95, 8686-

1404 Raabe, M., Veniant, M.M., Sullivan, M.A., Zlot, C.H., Bjorkegren, J., Nielsen, L.B., Wong, J.S., Hamilton, R.L., and Young, S.G. (1999). Analysis of the role of microsomal triglyceride transfer protein in the liver of tissue-specific knockout mice. J Clin Invest 103, 1287-1298.

1407 Raag, R., Appelt, K., Xuong, N.H., and Banaszak, L. (1988). Structure of the lamprey yolk lipid1408 protein complex lipovitellin-phosvitin at 2.8 A resolution. J Mol Biol 200, 553-569.

1409 Rava, P., Athar, H., Johnson, C., and Hussain, M.M. (2005). Transfer of cholesteryl esters and phospholipids as well as net deposition by microsomal triglyceride transfer protein. J Lipid Res $46,1779-1785$.

1412 Rava, P., and Hussain, M.M. (2007). Acquisition of triacylglycerol transfer activity by microsomal 1413 triglyceride transfer protein during evolution. Biochemistry 46, 12263-12274. 
Rava, P., Ojakian, G.K., Shelness, G.S., and Hussain, M.M. (2006). Phospholipid transfer activity of microsomal triacylglycerol transfer protein is sufficient for the assembly and secretion of apolipoprotein B lipoproteins. J Biol Chem 281, 11019-11027.

1417 Read, J., Anderson, T.A., Ritchie, P.J., Vanloo, B., Amey, J., Levitt, D., Rosseneu, M., Scott, J., 1418 and Shoulders, C.C. (2000). A mechanism of membrane neutral lipid acquisition by the 1419 microsomal triglyceride transfer protein. J Biol Chem 275, 30372-30377.

1420 Rehberg, E.F., Samson-Bouma, M.E., Kienzle, B., Blinderman, L., Jamil, H., Wetterau, J.R., Aggerbeck, L.P., and Gordon, D.A. (1996). A novel abetalipoproteinemia genotype. Identification of a missense mutation in the $97-\mathrm{kDa}$ subunit of the microsomal triglyceride transfer protein that prevents complex formation with protein disulfide isomerase. J Biol Chem $271,29945-29952$.

Ricci, B., Sharp, D., O'Rourke, E., Kienzle, B., Blinderman, L., Gordon, D., Smith-Monroy, C., Robinson, G., Gregg, R.E., Rader, D.J., et al. (1995). A 30-amino acid truncation of the microsomal triglyceride transfer protein large subunit disrupts its interaction with protein disulfide-isomerase and causes abetalipoproteinemia. J Biol Chem 270, 14281-14285.

1429 Robl, J.A., Sulsky, R., Sun, C.Q., Simpkins, L.M., Wang, T., Dickson, J.K., Jr., Chen, Y., 1430 Magnin, D.R., Taunk, P., Slusarchyk, W.A., et al. (2001). A novel series of highly potent 1431 benzimidazole-based microsomal triglyceride transfer protein inhibitors. J Med Chem 44, 851856.

1433 Schindelin, J., Arganda-Carreras, I., Frise, E., Kaynig, V., Longair, M., Pietzsch, T., Preibisch, S., Rueden, C., Saalfeld, S., Schmid, B., et al. (2012). Fiji: an open-source platform for biological-image analysis. Nat Methods 9, 676-682.

1436 Schlegel, A., and Stainier, D.Y. (2006). Microsomal triglyceride transfer protein is required for yolk lipid utilization and absorption of dietary lipids in zebrafish larvae. Biochemistry 45, 15179143815187.

1439 Schumaker, V.N., Phillips, M.L., and Chatterton, J.E. (1994). Apolipoprotein B and low-density 1440 lipoprotein structure: implications for biosynthesis of triglyceride-rich lipoproteins. Adv Protein 1441 Chem 45, 205-248.

1442 Sellers, J.A., Hou, L., Athar, H., Hussain, M.M., and Shelness, G.S. (2003). A Drosophila microsomal triglyceride transfer protein homolog promotes the assembly and secretion of human apolipoprotein B. Implications for human and insect transport and metabolism. J Biol 1445 Chem 278, 20367-20373.

1446 Sharp, D., Blinderman, L., Combs, K.A., Kienzle, B., Ricci, B., Wager-Smith, K., Gil, C.M., 1447 Turck, C.W., Bouma, M.E., Rader, D.J., et al. (1993). Cloning and gene defects in microsomal 1448 triglyceride transfer protein associated with abetalipoproteinaemia. Nature 365, 65-69.

1449 Shoulders, C.C., Brett, D.J., Bayliss, J.D., Narcisi, T.M., Jarmuz, A., Grantham, T.T., Leoni, 1450 P.R., Bhattacharya, S., Pease, R.J., Cullen, P.M., et al. (1993). Abetalipoproteinemia is caused 1451 by defects of the gene encoding the $97 \mathrm{kDa}$ subunit of a microsomal triglyceride transfer protein. 1452 Hum Mol Genet 2, 2109-2116. 

predicting effects of amino acid substitutions on proteins. Nucleic Acids Res 40, W452-457.

1455 Thierer, J.H., Ekker, S.C., and Farber, S.A. (In Press). LipoGlo: A sensitive and specific reporter 1456 of atherogenic lipoproteins. Nature Communications.

1457 Thompson, J.R., and Banaszak, L.J. (2002). Lipid-protein interactions in lipovitellin.

1458 Biochemistry 41, 9398-9409.

1459 Van der Auwera, G.A., Carneiro, M.O., Hartl, C., Poplin, R., Del Angel, G., Levy-Moonshine, A., 1460 Jordan, T., Shakir, K., Roazen, D., Thibault, J., et al. (2013). From FastQ data to high confidence variant calls: the Genome Analysis Toolkit best practices pipeline. Curr Protoc Bioinformatics 43, $111011-33$.

1463 Vernier, J., and Sire, M. (1977). Lipoprotéines de très basse densité et glycogène dans le syncytium vitellin, l'épithélium intestinal et le foie, aux stades précoces du développement embryonnaire chez la truite arc-en-ciel. Biol. cell 29, 45-54.

1466 Walsh, M.T., Di Leo, E., Okur, I., Tarugi, P., and Hussain, M.M. (2016). Structure-function analyses of microsomal triglyceride transfer protein missense mutations in abetalipoproteinemia and hypobetalipoproteinemia subjects. Biochim Biophys Acta 1861, 1623-1633.

Walsh, M.T., and Hussain, M.M. (2016). Targeting Microsomal Triglyceride Transfer Protein and Lipoprotein Assembly to Treat Homozygous Familial Hypercholesterolemia. Crit Rev. Clin. Lab Sci 54, 26-48. lipoprotein assembly to treat homozygous familial hypercholesterolemia. Crit Rev Clin Lab Sci 54, 26-48.

Walsh, M.T., Iqbal, J., Josekutty, J., Soh, J., Di Leo, E., Ozaydin, E., Gunduz, M., Tarugi, P., and Hussain, M.M. (2015). Novel Abetalipoproteinemia Missense Mutation Highlights the Importance of the N-Terminal beta-Barrel in Microsomal Triglyceride Transfer Protein Function. Circ Cardiovasc Genet 8, 677-687.

1479 Walther, T.C., Chung, J., and Farese, R.V., Jr. (2017). Lipid Droplet Biogenesis. Annu Rev Cell 1480 Dev Biol 33, 491-510.

1481 Walzer, C., and Schonenberger, N. (1979a). Ultrastructure and cytochemistry of the yolk 1482 syncytial layer in the alevin of trout (Salmo fario trutta L. and Salmo gairdneri R.) after hatching. 1483 II. The cytoplasmic zone. Cell Tissue Res 196, 75-93.

1484 Walzer, C., and Schonenberger, N. (1979b). Ultrastructure and cytochemistry study of the yolk 1485 syncytial layer in the alevin of trout (Salmo fario trutta L.) after hatching. I. The vitellolysis zone. 1486 Cell Tissue Res 196, 59-73.

1487 Wang, J., and Hegele, R.A. (2000). Microsomal triglyceride transfer protein (MTP) gene 1488 mutations in Canadian subjects with abetalipoproteinemia. Hum Mutat 15, 294-295. 
Wang, Y., McLeod, R.S., and Yao, Z. (1997). Normal activity of microsomal triglyceride transfer protein is required for the oleate-induced secretion of very low density lipoproteins containing apolipoprotein B from McA-RH7777 cells. J Biol Chem 272, 12272-12278.

1492 Wetterau, J.R., Aggerbeck, L.P., Bouma, M.E., Eisenberg, C., Munck, A., Hermier, M., Schmitz, 1493 J., Gay, G., Rader, D.J., and Gregg, R.E. (1992). Absence of microsomal triglyceride transfer 1494 protein in individuals with abetalipoproteinemia. Science 258, 999-1001.

1495 Wetterau, J.R., Combs, K.A., Spinner, S.N., and Joiner, B.J. (1990). Protein disulfide isomerase is a component of the microsomal triglyceride transfer protein complex. J Biol Chem 265, 98009807.

Wetterau, J.R., Gregg, R.E., Harrity, T.W., Arbeeny, C., Cap, M., Connolly, F., Chu, C.H., George, R.J., Gordon, D.A., Jamil, H., et al. (1998). An MTP inhibitor that normalizes atherogenic lipoprotein levels in WHHL rabbits. Science 282, 751-754.

1501 Wetterau, J.R., and Zilversmit, D.B. (1984). A triglyceride and cholesteryl ester transfer protein 1502 associated with liver microsomes. J Biol Chem 259, 10863-10866.

1503 Wetterau, J.R., and Zilversmit, D.B. (1985). Purification and characterization of microsomal 1504 triglyceride and cholesteryl ester transfer protein from bovine liver microsomes. Chem Phys 1505 Lipids 38, 205-222.

1506 Wetterau, J.R., and Zilversmit, D.B. (1986). Localization of intracellular triacylglycerol and 1507 cholesteryl ester transfer activity in rat tissues. Biochim Biophys Acta 875, 610-617.

1508 White, R.J., Collins, J.E., Sealy, I.M., Wali, N., Dooley, C.M., Digby, Z., Stemple, D.L., Murphy, D.N., Billis, K., Hourlier, T., et al. (2017). A high-resolution mRNA expression time course of 1510 embryonic development in zebrafish. Elife 6.

1511 Wiegand, M.D. (1996). Composition, accumulation and utilization of yolk lipids in teleost fish. 1512 Reviews in fish biology and fisheries 6, 259-286.

1513 Wu, X., Zhou, M., Huang, L.S., Wetterau, J., and Ginsberg, H.N. (1996). Demonstration of a 1514 physical interaction between microsomal triglyceride transfer protein and apolipoprotein $B$ 1515 during the assembly of ApoB-containing lipoproteins. J Biol Chem 271, 10277-10281.

1516 Yaniv, K., Isogai, S., Castranova, D., Dye, L., Hitomi, J., and Weinstein, B.M. (2006). Live 1517 imaging of lymphatic development in the zebrafish. Nat Med 12, 711-716.

1518 Zeituni, E.M., Wilson, M.H., Zheng, X., Iglesias, P.A., Sepanski, M.A., Siddiqi, M.A., Anderson, 1519 J.L., Zheng, Y., and Farber, S.A. (2016). Endoplasmic Reticulum Lipid Flux Influences Enterocyte Nuclear Morphology and Lipid-dependent Transcriptional Responses. J Biol Chem $291,23804-23816$. 